\title{
DEUTERIUM AND THE LOCAL INTERSTELLAR MEDIUM: PROPERTIES FOR THE PROCYON AND CAPELLA LINES OF SIGHT ${ }^{1}$
}

\author{
JefFrey L. Linsky, ${ }^{2,3}$ Athanassios Diplas, ${ }^{4}$ Brian E. WoOd, ${ }^{3}$ Alexander Brown, ${ }^{3,5}$ \\ Thomas R. Ayres, ${ }^{5}$ and Blair D. Savage 6 \\ Received 1994 November 28 ; accepted 1995 March 27
}

\begin{abstract}
We present Goddard High-Resolution Spectrograph observations of the interstellar H I and $D_{\text {I }}$ Ly $\alpha$ lines and the $\mathrm{Mg}$ II and Fe II resonance lines formed along the lines of sight toward the nearby stars Procyon (3.5 pc, $\left.l=214^{\circ}, b=13^{\circ}\right)$ and Capella $\left(12.5 \mathrm{pc}, l=163^{\circ}, b=5^{\circ}\right)$. New observations of Capella were obtained at orbital phase 0.80 , when the radial velocities of the intrinsic Ly $\alpha$ emission lines of each star were nearly reversed from those of the previous observations at phase 0.26 (analyzed by Linsky et al.). Since the intrinsic Ly $\alpha$ line of the Capella system-the "continuum" against which the interstellar absorption is measured--has different shapes at phases 0.26 and 0.80 , we can derive both the intrinsic stellar profiles and the interstellar absorption lines more precisely by jointly analyzing the two data sets. We derive interstellar parameters from the simultaneous analysis of the two data sets as follows: $(\mathrm{D} / \mathrm{H})_{\mathrm{LISM}}=(1.60 \pm 0.09[+0.05,-0.10$ systematic error] $) \times 10^{-5}$, temperature $T=7000 \pm 500$ [ \pm 400 systematic error] $K$, and microturbulence $\xi=1.6 \pm 0.4$ [and \pm 0.2 systematic error] $\mathrm{km} \mathrm{s}^{-1}$. (All random errors determined in this paper are $\pm 2 \sigma$.)

For the analysis of the Procyon line of sight, we first assumed that the intrinsic Lya line profile is a broadened solar profile, but this assumption does not lead to a good fit to the observed D I line profile for any value of $\mathrm{D} / \mathrm{H}$. We then assumed that $(\mathrm{D} / \mathrm{H})_{\mathrm{LISM}}=1.6 \times 10^{-5}$, the same value as for the Capella line of sight, and we modified the broadened solar profile to achieve agreement between the simulated and observed line profiles. The resulting asymmetric intrinsic stellar line profile is consistent with the shapes of the scaled $\mathrm{Mg}$ II line profiles. We believe therefore that the Procyon data are consistent with $(\mathrm{D} / \mathrm{H})_{\mathrm{LISM}}=1.6 \times 10^{-5}$, but the uncertainty in the intrinsic $\mathrm{Ly} \alpha$ emission-line profile does not permit us to conclude that the $\mathrm{D} / \mathrm{H}$ ratio is constant in the local interstellar medium (LISM). The temperature and turbulence in the Procyon line of sight are $T=6900 \pm 80\left( \pm 300\right.$ systematic error) $\mathrm{K}$ and $\xi=1.21 \pm 0.27 \mathrm{~km} \mathrm{~s}{ }^{-1}$. These properties are similar to those of Capella, except that the gas toward Procyon is divided into two velocity components separated by $2.6 \mathrm{~km} \mathrm{~s}^{-1}$ and the Procyon line of sight has a mean neutral hydrogen density that is a factor of 2.4 larger than that of the Capella line of sight. This suggests that the first $5.3 \mathrm{pc}$ along the Capella line of sight lies within the local cloud and the remaining $7.2 \mathrm{pc}$ lies in the hot gas surrounding the local cloud.

We propose that $n_{\mathrm{HI}}=0.1065 \pm 0.0028 \mathrm{~cm}^{-2}$ be adopted for the neutral hydrogen density within the local cloud and that $\xi=1.21 \pm 0.27 \mathrm{~km} \mathrm{~s}^{-1}$ be adopted for the nonthermal motions. The existence of different second velocity components toward the nearby stars Procyon and Sirius provides the first glimpse of a turbulent cloudlet boundary layer between the local cloud and the surrounding hot interstellar gas. We speculate that what is often called "turbulence" may instead be velocity shear within the local cloud that is not a rigid comoving structure. We also derive gas phase abundances of iron and magnesium in the Procyon line of sight and the abundance of oxygen in the Capella line of sight.

Within the context of standard big bang nucleosynthesis, our observed value of $(\mathrm{D} / \mathrm{H})_{\text {LISM }}$ leads to $0.042 \leq$ $\Omega_{\mathrm{B}} h_{50}^{2} \leq 0.09$, depending on the assumed model for Galactic chemical evolution of deuterium. Our lower limit $(\mathrm{D} / \mathrm{H})_{\text {LISM }} \geq 1.41 \times 10^{-5}$ provides a hard lower limit to the primordial $\mathrm{D}$ abundance and thus a hard upper limit on $\Omega_{\mathrm{B}} h_{50}^{2} \leq 0.125$. These limits are independent of Galactic chemical evolution models and only assume that $D$ is destroyed with time.
\end{abstract}

Subject headings: ISM : abundances - stars : individual ( $\alpha$ Canis Minoris, $\alpha$ Aurigae) - ultraviolet: stars

\section{INTRODUCTION}

The Hubble expansion, microwave background, and lightelement abundances are the main observational pillars upon which the standard big bang cosmology now rests. Of the three, the light-element abundances provide the main constraint on the total baryon density (both luminous and dark

\footnotetext{
1 Based on observations with the NASA/ESA Hubble Space Telescope, obtained at the Space Telescope Science Institute, which is operated by the Association of Universities for Research in Astronomy. Inc, under NASA contract NAS 5-26555.

${ }^{2}$ Staff Member, Quantum Physics Division, National Institute of Standards and Technology.
}

baryonic matter). The $\mathrm{D} / \mathrm{H}$ ratio is the most stringent of these constraints because (1) there are no known significant sources of deuterium after about $10^{3} \mathrm{~s}$ in the early universe, (2) the subsequent destruction of $\mathrm{D}$ is only by nuclear reactions in the cores of stars where D is the most fragile species, and (3) there is a steep monotonic slope between the primordial $\mathrm{D} / \mathrm{H}$ ratio

\footnotetext{
${ }^{3}$ Joint Institute for Laboratory Astrophysics, University of Colorado and National Intitute of Standards and Technology, Boulder, CO 80309-0440.

${ }^{4}$ CASS, University of California at San Diego.

${ }_{5}$ Center for Astrophysics and Space Astronomy, University of Colorado, Boulder, CO 80309-0389.

'Astronomy Department, University of Wisconsin, Madison, WI 53706.
} 
and the baryonic density in contemporary big bang nucleosynthesis models (e.g., Walker et al. 1991). Since none of the other light elements $\left({ }^{3} \mathrm{He},{ }^{4} \mathrm{He},{ }^{6} \mathrm{Li},{ }^{7} \mathrm{Li}, \mathrm{Be}\right.$, or B) share these properties, their abundances provide less certain estimates of the baryon density of the universe.

The importance of measuring $\mathrm{D} / \mathrm{H}$ has led to searches in a wide variety of environments including terrestrial seawater (using $\mathrm{HDO} / \mathrm{H}_{2} \mathrm{O}$ ), atmospheres of the giant planets (using $\mathrm{HD} / \mathrm{H}_{2}$ and $\mathrm{NH}_{2} \mathrm{D} / \mathrm{NH}_{3}$ ), cold interstellar clouds (using deuterated molecules and the as-yet-unsuccessful search for the 92 $\mathrm{cm}$ hyperfine transition of atomic D), and warm interstellar gas (using the Lyman series lines of $\mathrm{D}_{\mathrm{I}}$ and $\mathrm{H}_{\mathrm{I}}$ ). This work has been reviewed by Boesgaard \& Steigman (1985) and most recently by Wilson \& Rood (1994). Deuterated molecules have been observed in many sources, but their abundances relative to the corresponding undeuterated molecules are generally larger (often by orders of magnitude) than expected for sensible values of $\mathrm{D} / \mathrm{H}$. The overabundance usually is explained by the large mass difference between $D$ and $H$, the slightly larger binding energy of the deuterated molcules, and the cold environments in which they formed (e.g., Geiss \& Reeves 1981).

Determining the ratio of the column density of $D_{1}$ to that of $\mathrm{H} \mathrm{I}$ in warm interstellar gas $(T \approx 7000 \mathrm{~K})$, by measuring the absorption in the Lyman series lines along the lines of sight toward stars, is now thought to be the most accurate method for inferring the $\mathrm{D} / \mathrm{H}$ ratio in the Galaxy. Although the interstellar gas has been chemically processed over the lifetime of the Galaxy and the $\mathrm{D} / \mathrm{H}$ ratio must therefore be lower than primordial, this method should not suffer from other systematic errors. In particular, the relative ionization fraction, molecular association fraction, and degree of condensation onto dust grains should be the same for $D$ and $H$ in this environment. For Galactic lines of sight, only the Ly $\alpha$ line can be studied by the International Ultraviolet Explorer (IUE) and the Hubble Space Telescope (HST), but the higher. Lyman lines were observed toward a few hot stars by Copernicus. Unfortunately, $\mathrm{H}_{\mathrm{I}}$ absorption overlaps the $\mathrm{D}_{\mathrm{I}}$ line $(-0.3307$ A or $-81.55 \mathrm{~km} \mathrm{~s}^{-1}$ from the $\mathrm{H}$ I Ly $\alpha$ line). This overlap limits the use of the Ly $\alpha$ line to relatively nearby stars where the $\mathrm{H}_{\mathrm{I}}$ lines is not too opaque at the center of the D I line $\left(\log N_{\mathrm{H}_{1}}<\right.$ 18.7). A reanalysis of the best available Copernicus and IUE data led McCullough (1992) to estimate that the mean value of $\mathrm{D} / \mathrm{H}$ by number in the local interstellar medium (LISM) is
$(\mathrm{D} / \mathrm{H})_{\text {LiSM }}=1.5 \pm 0.2 \times 10^{-5}$. Unfortunately, these data have a relatively low signal-to-noise ratio $(\mathrm{S} / \mathrm{N})$, the $\mathrm{H}$ line is very saturated, and the intrinsic shapes of the $D_{I}$ and $H_{1}$ lines are unresolved at the spectral resolutions of IUE and Copernicus. We therefore initiated an HST observing program to obtain accurate values of $(D / H)_{\text {LISM }}$, to estimate the destruction of $D$ over the lifetime of the Galaxy and to infer the primordial $\mathrm{D} / \mathrm{H}$ ratio.

Since 1990 the Goddard High Resolution Spectrograph (GHRS) on the Hubble Space Telescope has been acquiring ultraviolet spectra with unprecedented spectral resolution and S/N. (See Brandt et al. 1994; Heap et al. 1995; and Soderblom 1993 for a description of the GHRS and its in-orbit capabilities at the time of these observations, prior to the installation of COSTAR.) The high spectral resolution and $S / N$ of these data permit us to measure very accurate column densities of interstellar $D_{I}, H_{I}$, and the heavier elements, together with the thermal and dynamic properties of interstellar gas, in the region of space near the Sun that includes the local warm cloud and surrounding hot substrate plasma and additional warm clouds. (See Vidal-Madjar et al. 1978; Lallement \& Bertin 1992; Frisch 1994a, b; Linsky et al. 1993 [hereafter Paper I]; and below for a discussion of the LISM properties.) In this second paper in a series, we reanalyze the Capella line of sight in $\S 2$ using observations obtained at both orbital quadratures, and in $\S 3$ we analyze observations of the Procyon line of sight. We discuss our results in $\S 4$ and present our conclusions in $\S 5$.

\section{THE CAPELLA LINE OF SIGHT}

\subsection{Previous Observations}

Our program to measure the $\mathrm{D} / \mathrm{H}$ ratio and the gas properties in the LISM began on 1991 April 15 with echelle observations of the resonance lines of $H_{\text {I }}$ and $D_{\text {I }}($ Ly $\alpha$ at $1216 \AA)$, the Fe II multiplet UV1 (at $2599 \AA$ ), and the Mg II and $k$ lines (at 2796 and $2803 \AA$ ) toward the Capella binary system. These and the subsequent GHRS observations of Capella (and Procyon) are summarized in Table 1. All the observations were obtained through the small science aperture (SSA) of the GHRS using the substep patterns listed in Table 1 (see Soderblom 1993; Paper I). The first observations were made at orbital phase 0.26 , when the G8 III star in the Capella system had a radial

TABLE 1

SUMMARY OF GHRS OBSERVATIONS

\begin{tabular}{|c|c|c|c|c|c|c|}
\hline Grating & $\begin{array}{c}\text { Aperture } \\
\text { and Substep } \\
\text { Pattern }\end{array}$ & $\begin{array}{c}\text { Spectral } \\
\text { Range } \\
(\AA)\end{array}$ & $\begin{array}{c}\text { Spectral } \\
\text { Resolution } \\
\left(\mathrm{km} \mathrm{s}^{-1}\right)\end{array}$ & $\begin{array}{l}\text { Exposure } \\
\text { Time } \\
\text { (s) }\end{array}$ & $\begin{array}{l}\text { Start } \\
\text { Time } \\
\text { (UT) }\end{array}$ & $\begin{array}{l}\text { Important } \\
\text { Features }\end{array}$ \\
\hline \multicolumn{7}{|c|}{ Capella at Phase 0.26 (1991 Apr 15) } \\
\hline $\begin{array}{l}\text { EA-46 } \ldots \ldots \ldots \\
\text { EB-22 } \ldots \ldots \\
\text { EB-20 } \ldots \ldots\end{array}$ & $\begin{array}{l}\text { SSA } 9 \\
\text { SSA } 7 \\
\text { SSA } 7\end{array}$ & $\begin{array}{l}1211-1217 \\
2594-2606 \\
2793-2807\end{array}$ & $\begin{array}{l}3.57 \\
3.27 \\
3.54\end{array}$ & $\begin{array}{r}3917 \\
707 \\
707\end{array}$ & $\begin{array}{l}10: 04 \\
13: 37 \\
13: 23\end{array}$ & $\begin{array}{l}\mathrm{H} \text { I }+\mathrm{D} \text { I } \lambda 1216 \\
\mathrm{Fe} \text { II } \lambda 2599 \\
\mathrm{Mg} \text { ॥ } \lambda \lambda 2796,2803\end{array}$ \\
\hline \multicolumn{7}{|c|}{ Capella at Phase 0.80 (1993 Sep 19) } \\
\hline $\begin{array}{l}\text { G160M } \ldots \ldots \\
\text { EB-20 } \ldots \ldots\end{array}$ & $\begin{array}{l}\text { SSA } 5 \\
\text { SSA } 7\end{array}$ & $\begin{array}{l}1206-1243 \\
2792-2807\end{array}$ & $\begin{array}{l}15 \\
3.54\end{array}$ & $\begin{array}{l}979 \\
707\end{array}$ & $\begin{array}{l}11: 11 \\
12: 48\end{array}$ & $\begin{array}{l}\mathrm{H} \text { I }+\mathrm{D}_{\mathrm{I}} \lambda 1216 \\
\mathrm{Mg} \text { I } \lambda \lambda 2796,2803\end{array}$ \\
\hline \multicolumn{7}{|c|}{ Procyon (1992 Dec 21) } \\
\hline $\begin{array}{l}\text { G160M } \ldots \ldots \\
\text { EB-20 } \ldots \ldots \\
\text { EB-22 } \ldots \ldots\end{array}$ & $\begin{array}{l}\text { SSA } 5 \\
\text { SSA } 7 \\
\text { SSA } 7\end{array}$ & $\begin{array}{l}1206-1243 \\
2793-2808 \\
2594-2606\end{array}$ & $\begin{array}{l}15 \\
3.54 \\
3.27\end{array}$ & $\begin{array}{r}3264 \\
653 \\
653\end{array}$ & $\begin{array}{l}17: 31 \\
19: 22 \\
19: 47\end{array}$ & $\begin{array}{l}H_{1}+D_{1} \lambda 1216 \\
M g \text { ll } \lambda \lambda 2796,2803 \\
F e \text { is } \lambda 2599\end{array}$ \\
\hline
\end{tabular}




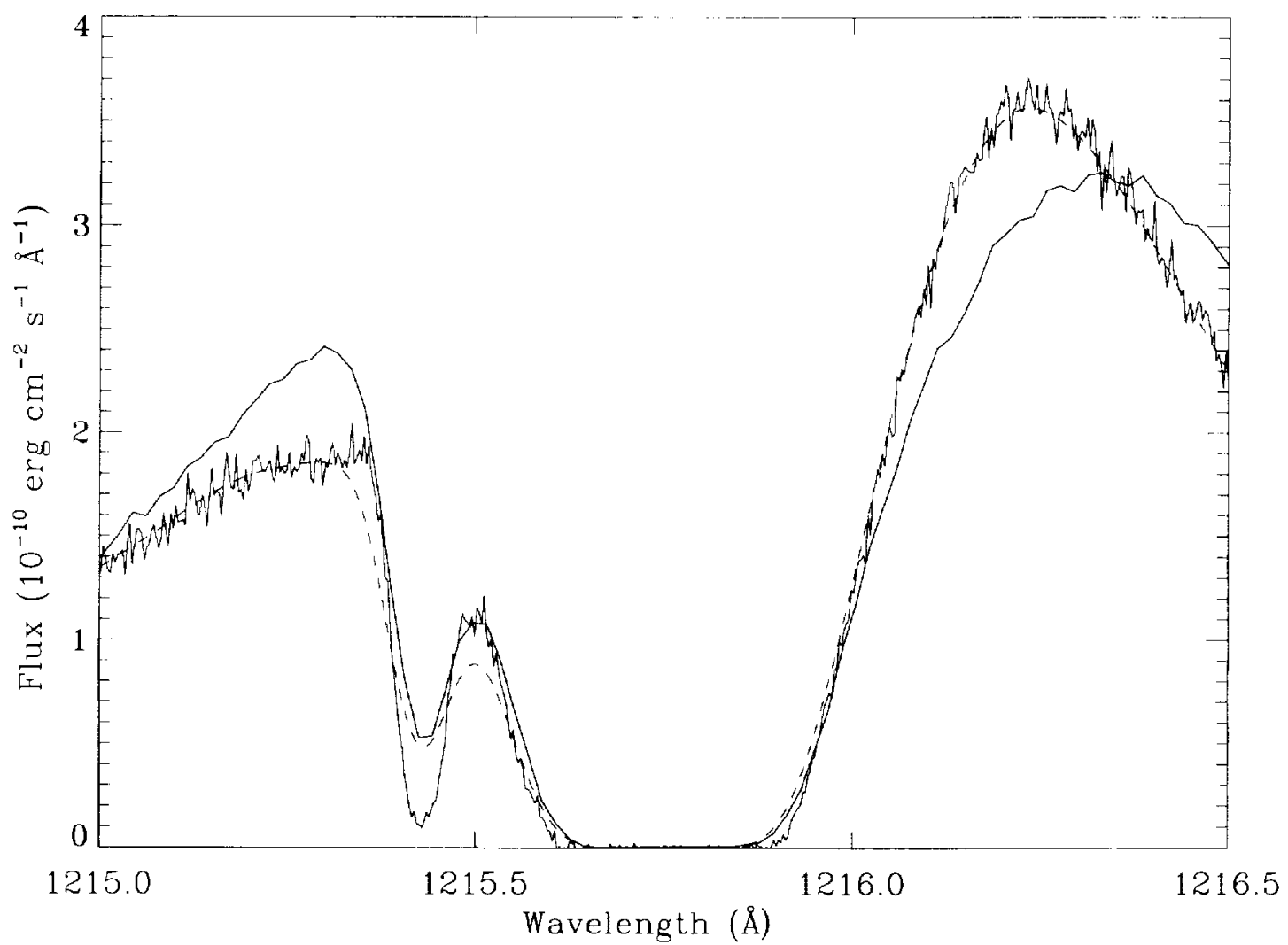

Fig. 1.-Ly $\alpha$ profiles of Capella at $\phi=0.26$ and 0.80 . Irregular solid line, high-dispersion echelle-A spectrum obtained 1991 April 15 at phase $\phi=0.26$. The profile consists of a broad $\mathrm{Ly} \alpha$ emission line produced in the chromospheres of both stars and absorption by interstellar $H$ I and $D$ I $(\Delta \lambda=-0.33 A$ relative to hydrogen) along the $12.5 \mathrm{pc}$ line of sight to Capella. Dashed line, $\phi=0.26$ spectrum smoothed to the moderate dispersion G160M resolution. Smooth solid line, moderate-dispersion G160M spectrum obtained 1993 September 19 at $\phi=0.80$. The difference between the smoothed $\phi=0.26$ spectrum and the $\phi=0.80$ spectrum outside of the saturated cores of the $\mathrm{H}$ I and $\mathrm{D}_{\mathrm{I}}$ lines is caused by the different radial velocities of the stellar emission lines at these two phases.

velocity of $+55.6 \mathrm{~km} \mathrm{~s}^{-1}$ and the G1 III star had a radial velocity of $+2.0 \mathrm{~km} \mathrm{~s}^{-1}$, very close to their maximum radial velocity separation. ${ }^{7}$ The broad $\mathrm{H}$ I interstellar absorption and narrower $D_{1}$ absorption are superposed upon Capella's chromospheric H I Ly $\alpha$ emission line (see Fig. 1). Detailed analysis of this spectrum and the $\mathrm{Fe}$ II and $\mathrm{Mg}$ II spectra in Paper I permitted us to obtain very accurate measurements of both the temperature $(T=7000 \pm 200 \mathrm{~K})$ and nonthermal broadening $\left(\xi=1.66 \pm 0.03 \mathrm{~km} \mathrm{~s}^{-1}\right)$ that characterize the gas in this line of sight and may be representative of the gas parameters in the local cloud. A careful analysis of the Capella spectra, including systematic errors associated with the uncertain intrinsic emission-line profile against which the interstellar absorption is measured, shows that the neutral hydrogen column density is $N_{\mathrm{HI}}=1.7-2.1 \times 10^{18} \mathrm{~cm}^{-2}$ and the number density ratio $(\mathrm{D} / \mathrm{H})_{\text {LISM }}=1.65 \quad(+0.07$, $-0.18) \times 10^{-5}$ for this line of sight.

\subsection{New Observations of Capella at Phase 0.80}

The largest identified systematic error in our previous analysis of the Capella phase 0.26 observations was the uncertain intrinsic Ly $\alpha$ emission-line profiles of the two stars in the Capella system, especially those parts of the emission lines within $0.5 \AA$ of line center but outside the dark core of the interstellar $\mathrm{H}_{\mathrm{i}} \mathrm{Ly} \alpha$ absorption line. These parts of the intrinsic

\footnotetext{
T We use the ephemeris of Ayres $(1984,1988)$, in which phase 0.0 is in conjunction with the slightly more massive G8 III star in front.
}

line profiles are critically important because they form the "continuum" against which the observed absorption-line profile is compared to determine the interstellar column densities and broadening parameters for $\mathrm{H} I$ and $D$ I. It was difficult to solve for both the shape of the two intrinsic stellar Ly $\alpha$ emission lines and the interstellar gas properties with spectra obtained at only one orbital phase, although $I U E$ spectra at other phases were helpful (Ayres et al. 1993). We therefore reobserved Capella on 1993 September 19 at orbital phase 0.80 , close to the opposite orbital quadrature (phase 0.75 ). Our goal was to verify the results in Paper I by analyzing the (assumed constant) interstellar absorption against the background of a somewhat different intrinsic emission line from the Capella system. At phase 0.80 the radial velocities of the G8 III and G1 III stars were +4.7 and $+55.5 \mathrm{~km} \mathrm{~s}^{-1}$, respectively, nearly the reverse of their velocities at phase 0.26 . Our observations (see Table 1) consisted of a G160M spectrum of the $L y \alpha$ region (see Fig. 1) and an echelle spectrum of the $\mathrm{Mg}$ II region (see Fig. 8 in Linsky et al. 1995). The echelle-A grating on side 1 of the GHRS was not operational at the time of these observations. These observations were obtained in the same way as described in Paper I, except that only the G160M spectrum was obtained with the FP-SPLIT procedure to reduce the photocathode fixed pattern noise and granularity. The onboard Doppler compensation to correct for the changing spacecraft radial velocity was enabled during all observations.

The processing of the phase 0.80 spectra and reprocessing of the phase 0.26 spectra employed the 1993 December version of 
the CALHRS calibration software at the GHRS computing facility at the Goddard Space Flight Center. The processing includes converting raw counts to count rates and correcting for paired pulse events and diode-to-diode nonuniformities. The quarter diode stepped spectra were merged to form the individual spectra of 2000 wavelength samples. The final reduction includes proper alignment of the individual FPSPLIT spectra (using the HRS MERGE procedure), rejection of fixed pattern noise structures, correction for scattered light and the radiation background, and wavelength calibration including the effects of thermal drifts. For the echelle-B measurements, the background correction was determined as described in Paper I. Wavelength calibration using the CALHRS reduction software as of 1993 December was performed with the special platinum lamp calibration exposures obtained immediately before each stellar integration.

In Figure 1 we compare the observed Ly $\alpha$ line at phase 0.80 with the phase 0.26 profile degraded to the resolution of the G160M grating. The two profiles are nearly identical in the cores of the $\mathrm{H}_{\mathrm{I}}$ and $\mathrm{D}_{\mathrm{I}}$ lines where the interstellar optical depth is large and the shape of the underlying stellar emission line is relatively unimportant, but the two profiles differ greatly where the interstellar opacity is small, including spectral

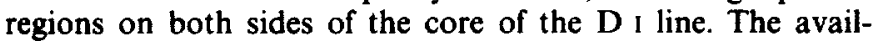
ability of data at both quadratures makes it feasible to determine both the shapes of the stellar emission-line profiles and the interstellar absorption parameters.

\subsection{Combined Analysis of the Phase 0.26 and 0.80 Capella Spectra}

Our analysis of both Capella data sets differs from that used in the previous analysis of the phase 0.26 data in Paper I in two important respects. First, we developed models for the intrinsic Ly $\alpha$ emission-line profiles of the G1 III and G8 III stars (see Fig. 2) and required that they be invariant with phase, aside from the different orbital velocity shifts. This requirement is sensible, since Ayres (1984) and Wood \& Ayres (1995) have detected no significant changes in the Capella ultraviolet emission-line fluxes with orbital phase or time at the $\pm 5 \%$ sensitivity level of $I U E$. During the iterative fitting process, we modified the interstellar parameters and the intrinsic line profiles for each star, although the changes from the intrinsic profiles used in Paper I are small, to minimize the value of $\chi^{2}$ between the observed and simulated Ly $\alpha$ line profiles at both phases.

A second difference from our original analysis in Paper $I$ is the use of the two-component instrumental point spread function (PSF) for the echelle data described by Spitzer \& Fitzpatrick (1993). It consists of a narrow Gaussian "core" with FWHM $=1.05$ diodes and a broader Gaussian "halo" with FWHM $=5.0$ diodes. The relative strengths of these two components depend on wavelength (Cardelli, Ebbets, \& Savage 1990). We do not explicitly include the third component of the PSF measured by Cardelli et al.-the broad power-law

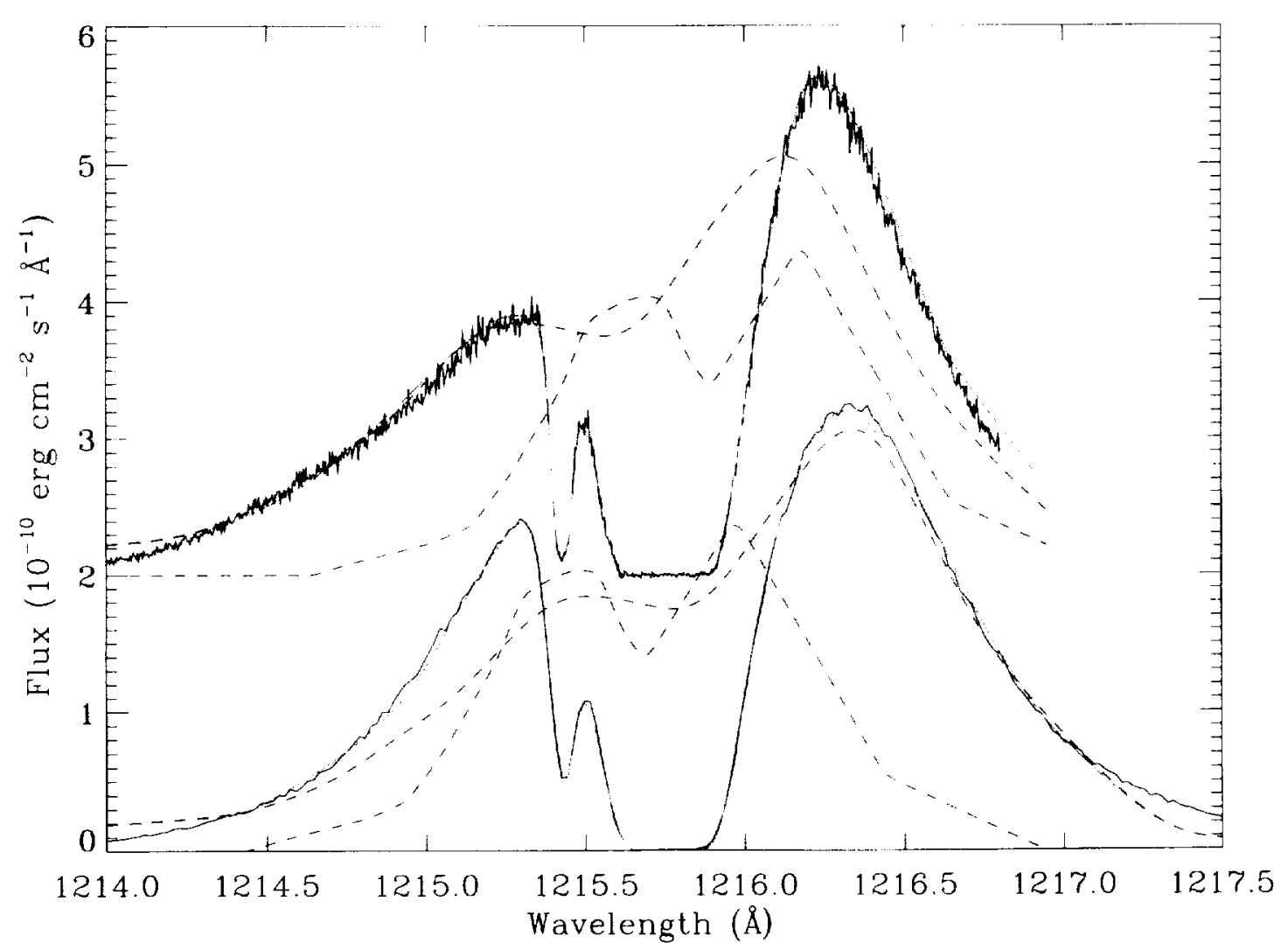

FiG. 2. Capella at $\phi=0.26$ and 0.80 . Upper graph: High-dispersion $(\lambda / \Delta \lambda=84,000)$ spectrum (displaced upward by two units) of the $1216 \mathrm{~A}$ region of Capella at $\phi=0.26$ obtained with the echelle-A grating. Dashed lines are the assumed intrinsic emission-line profiles of the two stars in the Capella system. Thin solid line is the sum of the two intrinsic profiles folded through the interstellar medium with the parameters listed in Table 2. Lower graph: Moderate-dispersion $(\lambda / \Delta \lambda=20.000)$ spectrum of the $1216 \mathrm{~A}$ region of Capella at $\phi=0.80$ obtained with the $G 160 \mathrm{M}$ grating. Dashed lines are the same intrinsic line profiles, but with the radial velocities of each star at this phase. 
"wing"-but instead, following Spitzer \& Fitzpatrick, we subtract the measured flux in the saturated core of the interstellar Ly $\alpha$ line from the entire line profile. In Paper I we used a single Gaussian PSF with an FWHM $=3.57 \mathrm{~km} \mathrm{~s}^{-1}$ corresponding to 1.20 diodes at Ly $\alpha$. We find that the resulting changes in the line width parameter $b$ for all the lines in our reanalysis of the phase 0.26 data set lie within the errors of our initial analysis (Paper I) and our reanalysis. The parameter $b$ has its usual definition $b^{2}=(2 k T / m)+\xi^{2}$, where $\xi$ is the most probable speed of the turbulent motions and $m$ is the atomic mass.

Figure 8 in Linsky et al. (1995) shows the echelle spectra of the Mg if $\lambda \lambda 2796,2803$ lines at the opposite orbital phases and the intrinsic emission-line profiles derived in our analysis. Because the interstellar absorption features are narrow and the $\mathrm{S} / \mathrm{N}$ of the data is very high, we used a polynomial interpolation between the emission-line peaks on either side of the interstellar absorption to produce a smooth and, we believe, very accurate "continuum" against which to measure the interstellar absorption. Figure 3 compares the residual intensities at phase 0.80 for the $\mathrm{Mg}$ II lines, obtained by dividing the observed line profiles by the sum of the intrinsic profiles of the two stars; the computed residual intensities using the interstellar parameters are listed in the third column of Table 2. (All random errors determined in this paper are $\pm 2 \sigma$.) The $\mathrm{Mg}$ II column densities, velocities, and broadening parameters derived from the data at phase 0.80 and rederived from the data at phase 0.26 are very close to those determined in Paper I for the observations at phase 0.26 .

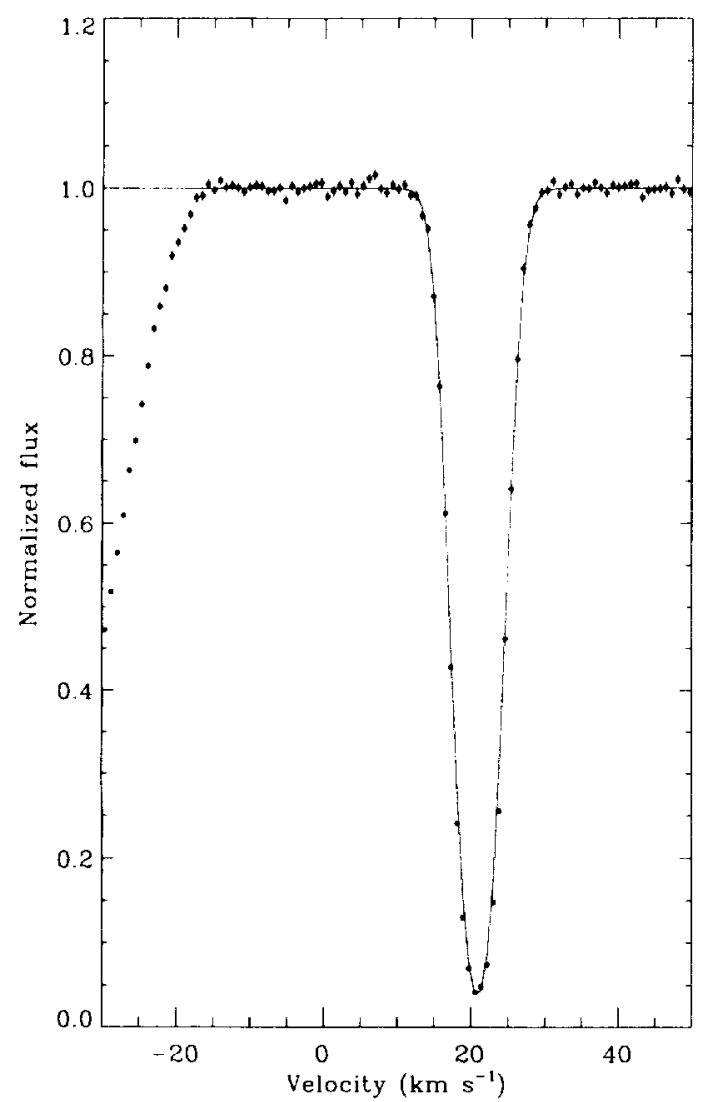

We next reanalyzed the phase $0.26 \mathrm{Ly} \alpha$ profiles by using the two-component PSF described above. The observed and simulated Ly $\alpha$ profiles for phase 0.26 are compared in Figure 2 through the use of the interstellar parameters listed in Table 2. The simulated and observed profiles are in excellent agreement, but the LISM parameters differ slightly from those obtained in the original analysis of the phase 0.26 data. We then analyzed the lower resolution phase 0.80 Ly $\alpha$ profiles obtained with the $\mathrm{G} 160 \mathrm{M}$ grating. The resulting parameters for the $\mathrm{H}$ I and D I column densities and interstellar parameters are in excellent agreement with those obtained from the higher resolution echelle spectra at phase 0.26 , except for the temperature which may be a result of inadequate resolution of the D I line. This gives us confidence that the lower resolution G160M spectrum did not seriously degrade the quality of the final results. We believe that the small differences in the interstellar parameters between the original analysis of the phase 0.26 echelle spectrum and the present reanalysis are a result of both the different PSF used and the small differences in the assumed intrinsic stellar emission lines.

Our analysis of the phase 0.80 data and reanalysis of the phase 0.26 data are in excellent agreement with the same temperature for the LISM $(T=7000 \pm 500 \mathrm{~K}$ compared to the previous result of $7000 \pm 200 \mathrm{~K})$ and a similar value for the turbulent velocity $\left(\xi=1.6 \pm 0.4 \mathrm{~km} \mathrm{~s}^{-1}\right.$ compared to the previous result of $\xi=1.66 \pm 0.03 \mathrm{~km} \mathrm{~s}^{-1}$ ). We estimate that $(\mathrm{D} / \mathrm{H})_{\text {LISM }}=1.60 \pm 0.09 \times 10^{-5}$ for the Capella line of sight, where the uncertainty is taken to be the difference between the

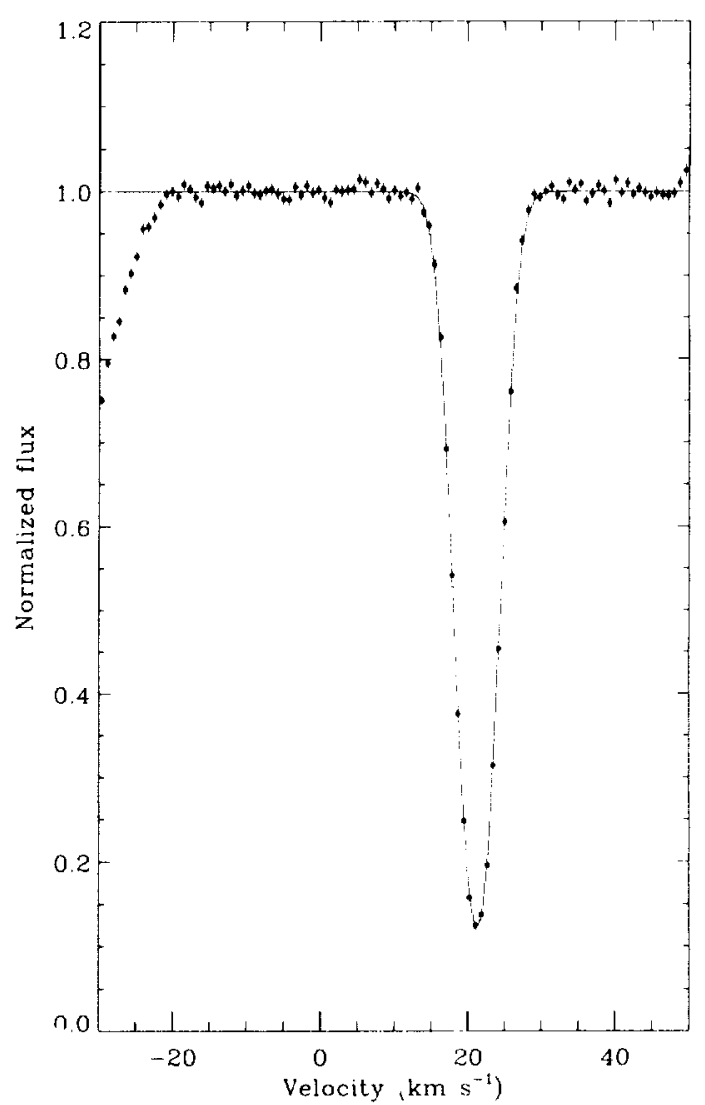

Fig. 3. - Capella at $\phi=0.80$ for $\mathrm{Mg}$ II $k$ and $\mathrm{Mg}$ II $h$. Profiles of the interstellar $\mathrm{Mg}$ II $k$ (left) and $h$ (right) lines (dots with $\pm 1 \sigma$ error bars) at $\phi=0.80$ after dividing by the sum of the assumed intrinsic line profiles of the two stars. Solid lines are simulated interstellar line profiles for the parameters listed in Table 2. 
TABLE 2

Interstellar Parameters for Capella and Procyon lines of Sight

\begin{tabular}{|c|c|c|c|c|}
\hline \multirow[b]{2}{*}{ Parameter } & \multicolumn{2}{|c|}{ Capella } & \multicolumn{2}{|c|}{ ProCYON* } \\
\hline & phase 0.26 & phase 0.80 & broadened $\odot$ & $(\mathrm{D} / \mathrm{H})_{\text {Copelli: }}$ \\
\hline $\mathrm{Mg}$ II $k v_{c}\left(\mathrm{~km} \mathrm{~s}^{-1}\right)$. & $23.04 \pm 0.10$ & $20.93 \pm 0.10$ & $21.53 \pm 0.10$ & $21.53 \pm 0.10$ \\
\hline $\mathrm{Mg}$ II $k b\left(\mathrm{~km} \mathrm{~s}^{-1}\right) \ldots$ & $2.64 \pm 0.13$ & uncertain & $3.13 \pm 0.16$ & $3.13 \pm 0.16$ \\
\hline $\mathrm{Mg}$ II $k N_{L}\left(10^{12} \mathrm{~cm}^{-2}\right)$. & $6.44 \pm 0.32$ & uncertain & $3.54 \pm 0.16$ & $3.54 \pm 0.16$ \\
\hline $\mathrm{Mg}$ II $h v_{c}\left(\mathrm{~km} \mathrm{~s}^{-1}\right) \ldots \ldots$ & $22.72 \pm 0.10$ & $21.27 \pm 0.10$ & $21.18 \pm 0.10$ & $21.18 \pm 0.10$ \\
\hline $\mathrm{Mg}$ is $h b\left(\mathrm{~km} \mathrm{~s}^{-1}\right) \ldots$ & $2.43 \pm 0.12$ & $2.51 \pm 0.12$ & $2.92 \pm 0.15$ & $2.92 \pm 0.15$ \\
\hline $\mathrm{Mg}$ If $h N\left(10^{12} \mathrm{~cm}^{-2}\right)$. & $7.03 \pm 0.35$ & $6.94 \pm 0.35$ & $3.23 \pm 0.17$ & $3.23 \pm 0.17$ \\
\hline $\mathrm{Fe} \| v_{c}\left(\mathrm{~km} \mathrm{~s}^{-1}\right) \ldots \ldots$ & $20.65 \pm 0.10$ & & $20.23 \pm 0.10$ & $20.23 \pm 0.10$ \\
\hline $\mathrm{Fe}$ II $b\left(\mathrm{~km} \mathrm{~s}^{-1}\right) \ldots \ldots$ & $2.36 \pm 0.12$ & $\ldots$ & $2.71 \pm 0.13$ & $2.71 \pm 0.13$ \\
\hline Fe If $N_{L}\left(10^{12} \mathrm{~cm}^{-2}\right)$ & $3.12 \pm 0.16$ & & $1.86 \pm 0.10$ & $1.86 \pm 0.10$ \\
\hline $\mathrm{H} \backslash v_{c}\left(\mathrm{~km} \mathrm{~s}^{-1}\right) \ldots \ldots$ & $21.74 \pm 0.10$ & $22.48 \pm 0.10$ & $22.00 \pm 0.10$ & $22.00 \pm 0.10$ \\
\hline $\mathrm{H}$ I $b\left(\mathrm{~km} \mathrm{~s}^{-1}\right)$ & $11.15 \pm 0.10$ & $10.59 \pm 0.15$ & $10.83 \pm 0.15$ & $10.95 \pm 0.10$ \\
\hline H I $N_{L}\left(10^{18} \mathrm{~cm}^{-2}\right)$ & $1.74 \pm 0.02$ & $1.73 \pm 0.02$ & $1.18 \pm 0.03$ & $1.14 \pm 0.02$ \\
\hline D I $v_{c}\left(\mathrm{~km} \mathrm{~s}^{-1}\right) \ldots$ & $22.04 \pm 0.10$ & $21.48 \pm 0.10$ & $22.00 \pm 0.10$ & $22.00 \pm 0.10$ \\
\hline $\mathrm{D} ; b\left(\mathrm{~km} \mathrm{~s}^{-1}\right) \ldots \ldots$ & $7.96 \pm 0.10$ & $7.58 \pm 0.10$ & $7.82 \pm 0.10$ & $7.91 \pm 0.10$ \\
\hline D I $N_{I}\left(10^{13} \mathrm{~cm}^{-2}\right)$ & $2.85 \pm 0.10$ & $2.71 \pm 0.10$ & $1.65 \pm 0.10$ & $1.82 \pm 0.10$ \\
\hline$T(\mathrm{~K}) \ldots \ldots \ldots \ldots \ldots$ & $7320 \pm 120$ & $6567 \pm 120$ & $6769 \pm 35$ & $6931 \pm 110$ \\
\hline$\xi\left(\mathrm{km} \mathrm{s}^{-1}\right) \ldots \ldots \ldots \ldots$ & $1.47 \pm 0.31$ & $1.65 \pm 0.20$ & $2.15 \pm 0.12$ & $2.13 \pm 0.13$ \\
\hline $\mathrm{D} / \mathrm{H}\left(\times 10^{-5}\right)$ & $1.64 \pm 0.05$ & $1.57 \pm 0.05$ & $1.40 \pm 0.20$ & $(1 . \overline{60})$ \\
\hline
\end{tabular}

- The $\mathrm{Mg}$ Il and $\mathrm{Fe}$ il parameters for Procyon assume a one-component interstellar medium.

two different determinations. These cited errors are random, since they represent the range in the parameters consistent with the noise of the data. The $\pm 2 \sigma$ uncertainties in $N_{\mathrm{H}}$ and $b_{\mathrm{H}}$ listed in columns (2) and (3) of Table 2 are derived from contour plots (e.g., Fig. 4) corresponding to different values of $\chi^{2}$ between the simulated and observed profiles. The increase in the errors for $T$ and $\xi$ compared to Paper I results from more detailed simulations and contour plots that demonstrate the tradeoff between thermal and nonthermal broadening that is consistent with the uncertainties in the measured $b$ values.

There are also systematic errors associated with the uncertain PSF and intrinsic stellar line profiles. We estimate these to be $\pm 400 \mathrm{~K}$ for the temperature and $\pm 0.2 \mathrm{~km} \mathrm{~s}^{-1}$ for the turbulent velocity values based on the differences between the

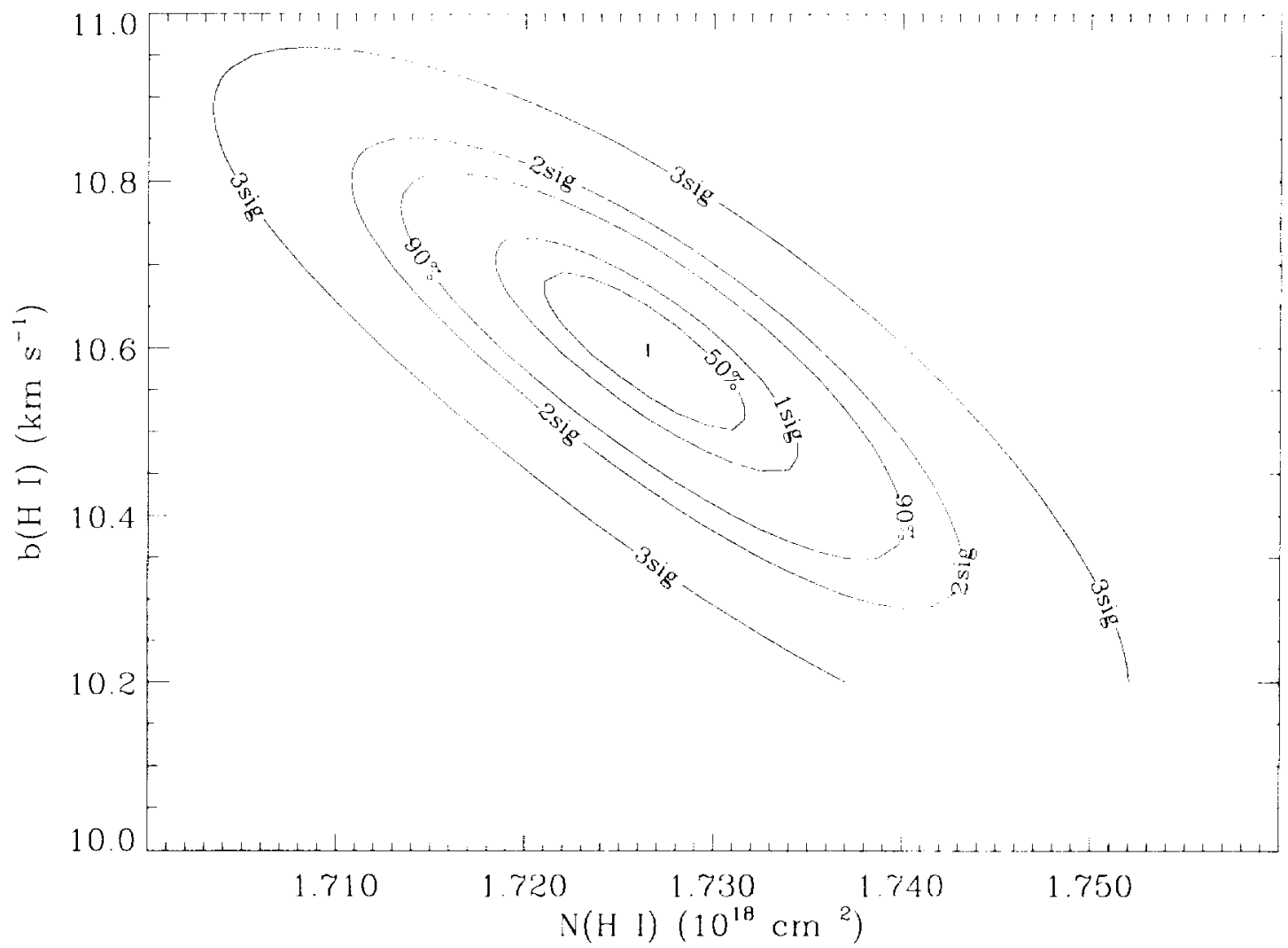

Fig. 4.- Contour plot of the $\mathrm{H}$ I column density $N_{\mathrm{H}}$, vs. hydrogen-broadening parameter $b_{\mathrm{H}}$ to fit the Capella data at $\phi=0.80$ 
TABLE 3

local Interstellar Medium Properties Toward Procyon and Capella

\begin{tabular}{|c|c|c|c|c|}
\hline$\frac{N_{\mathrm{HI}}}{\left(10^{17} \mathrm{~cm}^{-2}\right)}$ & $\left(\mathrm{km} \mathrm{s}^{-1}\right)$ & $\begin{array}{c}n_{111} \\
\left(\mathrm{~cm}^{-3}\right)\end{array}$ & $\begin{array}{c}\mathrm{D} / \mathrm{H} \\
\left(10^{-5}\right)\end{array}$ & References \\
\hline \multicolumn{5}{|c|}{ Procyon $\left(l=214^{\circ}, b=13^{\circ}, d=3.5 \mathrm{pc}\right)$} \\
\hline $10-14$ & $10-15$ & $0.09-0.13$ & $0.7-1.9$ & Anderson et at. 1978 \\
\hline $10-22$ & $3-15$ & $0.09-0.20$ & $\geq 0.8$ & Murthy et al. 1987 \\
\hline $11.2-11.8$ & $10.68-10.88$ & $0.104-0.109$ & $(1.6)$ & This paper \\
\hline \multicolumn{5}{|c|}{ Capella $\left(l=163^{\circ}, b=5^{\circ}, d=12.5 \mathrm{pc}\right)$} \\
\hline $9-15$ & 10 & $0.02-0.04$ & $2.2-9.6$ & Dupree, Baliunas, \& Shipman 1977 \\
\hline $15-19$ & $8-10$ & $0.04-0.05$ & $1.8-4.0$ & McClintock et al. 1978 \\
\hline $9-17$ & $3-12$ & $0.022-0.04$ & $\geq 2.0$ & Anderson 1979 \\
\hline $6-18$ & $13-17$ & $0.014-0.046$ & $2.4-6.0$ & Murthy et al. 1990 \\
\hline $17-21$ & $10.9-11.4$ & $0.044-0.055$ & $1.47-1.72$ & Linsky et al. 1993 \\
\hline $17.1-17.6$ & $10.44-11.25$ & $0.0443-0.0456$ & $1.41-1.74$ & This paper \\
\hline
\end{tabular}

derived interstellar parameters for the two different PSFs. The uncertainty in $\mathrm{D} / \mathrm{H}$ is smaller than that given in Paper I, since we now have data at two different phases with which to infer the intrinsic line profiles. We estimate the systematic error in $(\mathrm{D} / \mathrm{H})_{\text {LISM }}$ to be $(+0.05,-0.10) \times 10^{-5}$. Table 3 compares these interstellar gas properties with previous determinations for the Capella and Procyon lines of sight.

\section{THE PROCYON LINE OF SIGHT}

\subsection{Observations of Procyon}

Our second target was Procyon, an F5 IV-V star located 3.5 pc along a line of sight about $52^{\circ}$ from Capella. We observed the target on 1992 December 21 in the same way as we observed Capella at phase 0.26 , except that the Ly $\alpha$ line was observed through the small science aperture (SSA) with the G160M grating on side 2 . The spectral resolution at $\mathrm{Ly} \alpha$ was only $20,000\left(15 \mathrm{~km} \mathrm{~s}^{-1}\right)$ compared with $84,000\left(3.57 \mathrm{~km} \mathrm{~s}^{-1}\right)$ when we used the Echelle-A grating. The $\mathrm{Mg}$ II and $\mathrm{Fe}$ II lines were observed through the SSA with Echelle-B, providing the same high spectral resolution as for Capella. These observations were obtained and reduced in the same way as the phase 0.80 Capella observations, except that we used the 1993 September version of CALHRS.

Figure 5 illustrates the echelle spectra of the Procyon $\mathrm{Mg}$ II resonance lines. The broad absorption line wings are produced

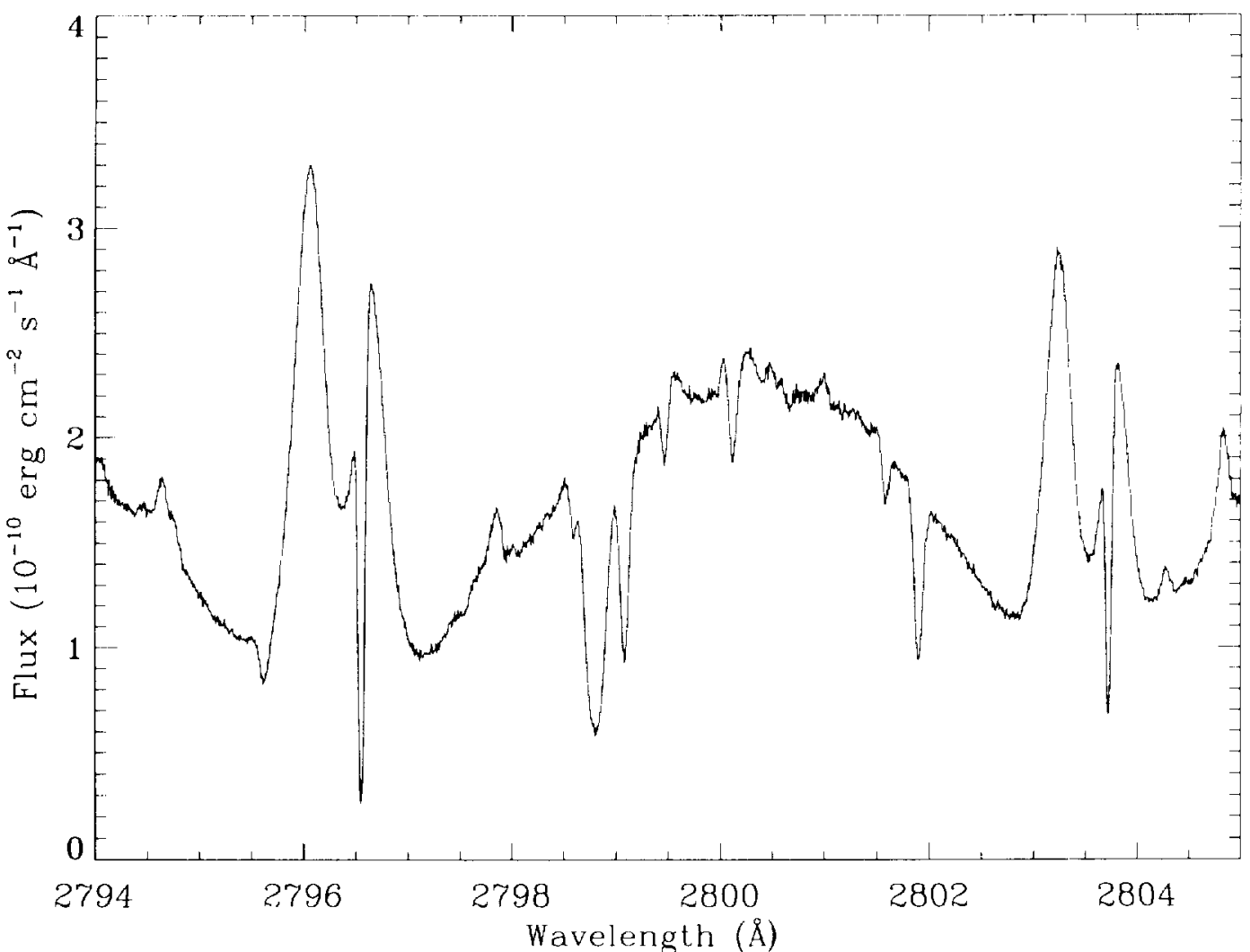

FIG. 5.-Echelle-B spectrum of Procyon including the Mg II lines. Narrow absorption lines to the right of the centers of each self-reversed emission line are inverstellar. 
by the optically thick Mg II lines in Procyon's photosphere. The bright emission features centered at 2796 and $2803 \AA$ are produced by collisionally excited $\mathrm{Mg}$ II in the warm chromosphere. The absorption features in the centers of the emission lines are self-reversals produced by $\mathbf{M g}$ II ions at the top of the chromosphere. Here the populations of the excited states are smaller than at the base of the chromosphere because of decreased collisional excitation at the lower densities and the lower line radiation field that occurs where the line becomes effectively thin. About $+0.2 \AA$ from the central absorption features of both lines are very narrow absorption features produced by $\mathrm{Mg}$ II ions in the interstellar medium. Figure 6 shows the photospheric Fe il $\lambda 2599$ absorption line upon which a narrow interstellar component also is superposed.

\subsection{Analysis of the Procyon Line of Sight}

Figures 7 and 8 illustrate the $\mathrm{Mg}$ Il and Fe II residual intensities obtained by dividing the observed absorption lines by an interpolated stellar emission-line profile. We initially assumed a single velocity component for the Procyon line of sight and deduced the interstellar $\mathrm{Mg}$ II and $\mathrm{F}$ II column densities, velocities, and broadening parameters that minimized the residuals of the fit. Figures 7 and 8 compare the observed and simulated absorption profiles for the one-component model of the LISM that minimizes $\chi^{2}$ of the residuals. The parameters for this model are given in column (4) of Table 2.

Careful inspection of Figure 7 shows good agreement between the observed and computed residual intensities on the long-wavelength side of all the lines but small systematic disagreements on the short-wavelength side of the lines. The best- fit one-component model predicts $\mathbf{M g}$ II residual fluxes that are too low at $20-21 \mathrm{~km} \mathrm{~s}^{-1}$ and too high at $14-17 \mathrm{~km} \mathrm{~s}^{-1}$. As shown in Figure 9, we are able to improve the fits to the data greatly (the reduced $\chi^{2}$ decreases from 3.2 to 1.3 ) with a twocomponent model in which the bulk velocities differ by $2.6 \mathrm{~km}$ $\mathrm{s}^{-1}$ (mean value for the $h$ and $k$ lines), slightly less than the instrument resolution of $3.54 \mathrm{~km} \mathrm{~s}^{-1}$. The parameters for the best-fit two-component model are given in Table 4 . About $64 \%$ of the $\mathrm{Mg}$ Il column density is in the component at lower radial velocity. The $\mathrm{Fe}$ II line data are too noisy to discriminate between the one- and two-component models (see Fig. 8).

Since the interstellar $\mathrm{H} \mathrm{I}$ absorption is saturated and broad, we must develop a detailed model for the intrinsic stellar Ly $\alpha$ line profile against which to measure the interstellar $\mathrm{H} I$ and D I absorption. Fortunately Procyon (unlike Capella) is similar to the Sun in its spectral type, low rotational velocity, and weak chromospheric emission. Thus, the intrinsic chromospheric line shape should be similar to that of the Sun. Since the Lyo lines of solar-type stars broaden with increasing luminosity (Landsman \& Simon 1993), we broadened the solar line profile (about line center) by a range of multiplicative factors to best match the observed line profile outside of the interstellar absorption. A broadening factor of 1.4, as shown in Figure 10, produces a good fit to the observed profile, except in the region of the $\mathrm{D}$ I line.

We have fitted the $H$ I and $D$ I lines with both onecomponent and two-component models for which the velocities and relative column densities of each component were assumed to be the same as for $\mathbf{M g}$ II. The resulting interstellar parameters and $H_{I}$ and $D_{I}$ column densities of the one-

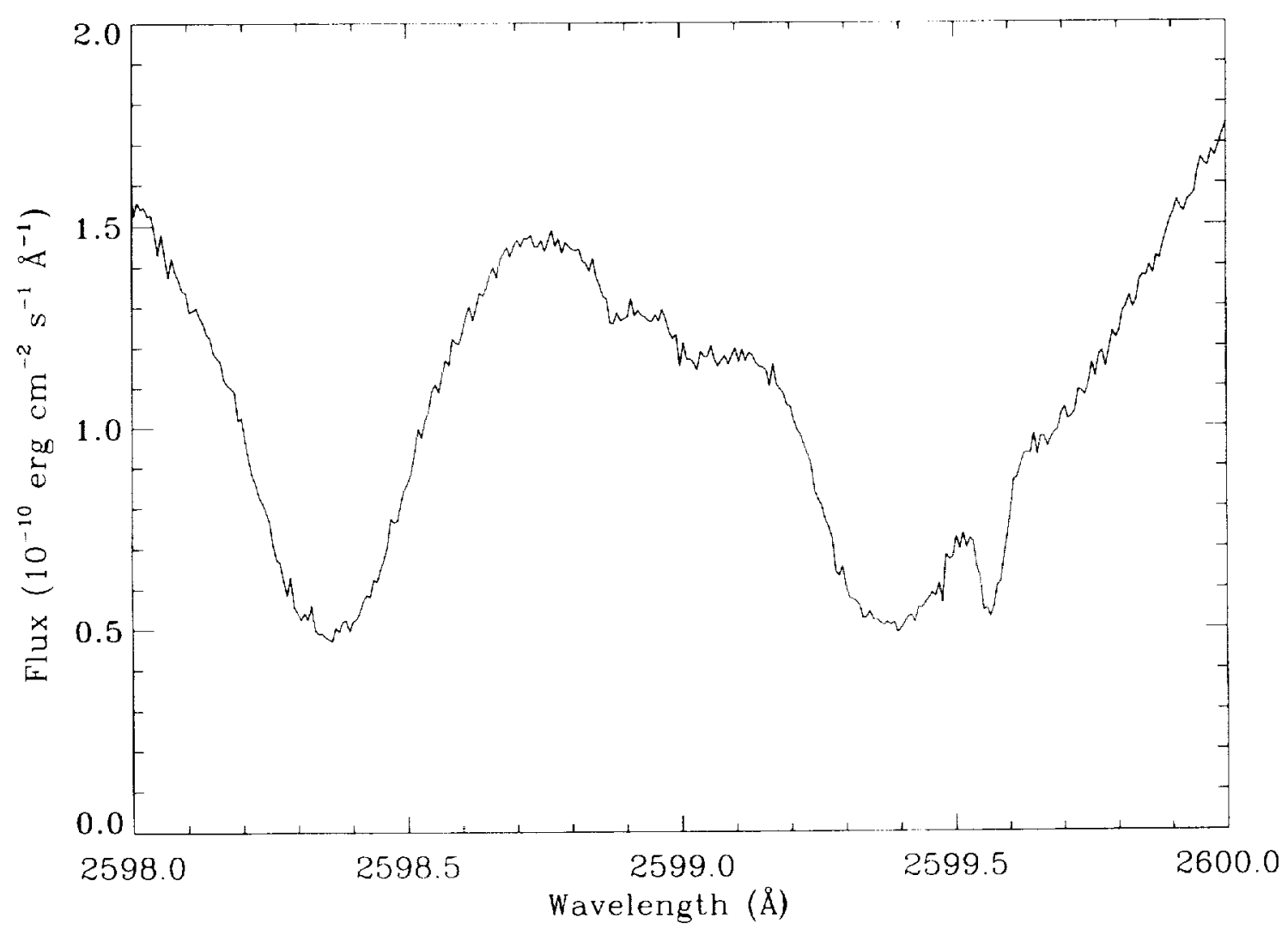

FIG. 6.-Echelle-B spectrum of Procyon including two Fe II resonance lines. Narrow absorption line at $2599.6 \AA$ is interstellar. 

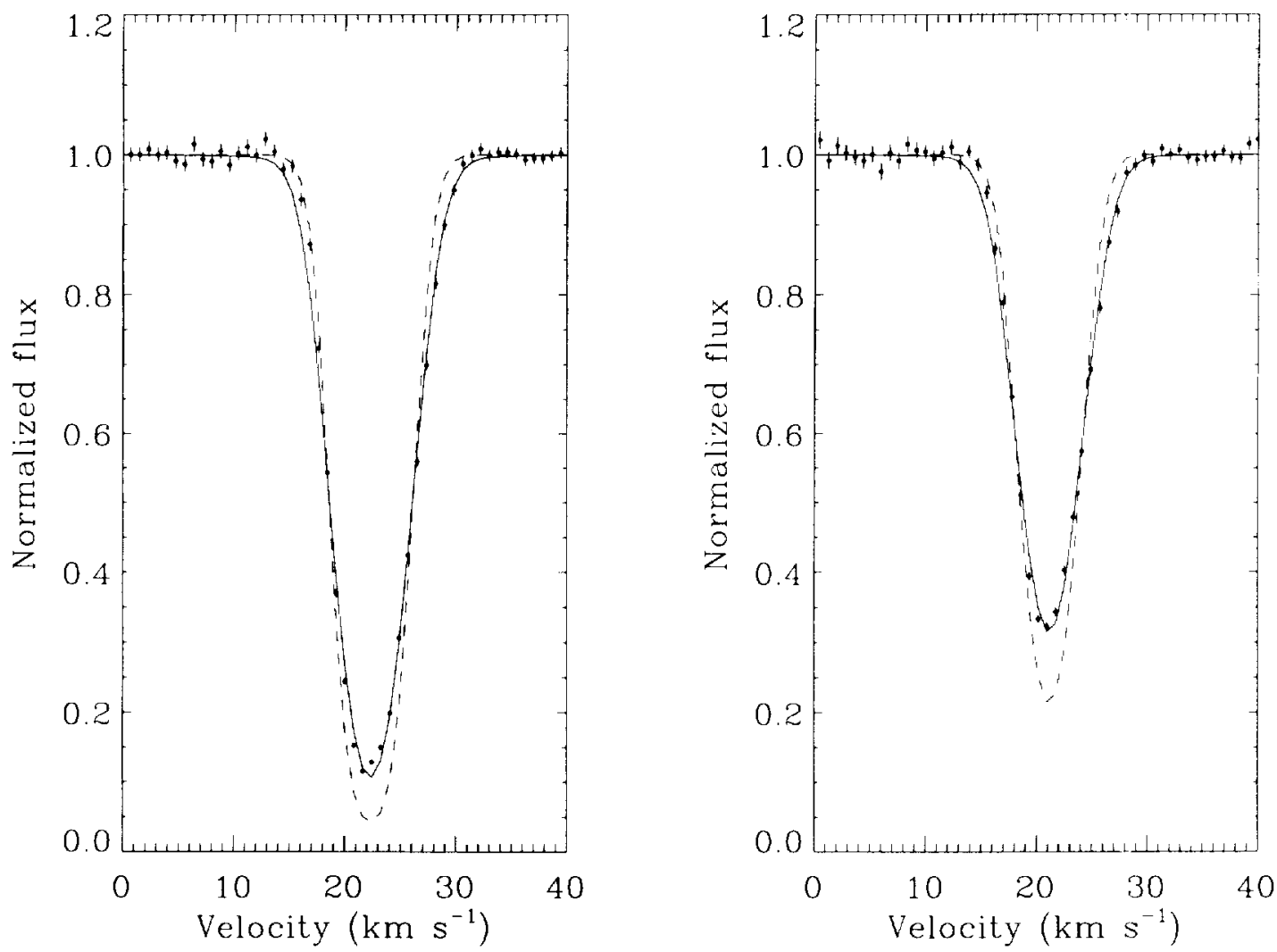

Fig. 7.-Echelle-B spectra of Procyon's normalized interstellar Mg $\| k(2796 \AA)(l e f t)$ and $h(2803 \AA)(r i g h t)$ lines (with $\pm 1 \sigma$ error bars): the profile that best fits the data using the single velocity component interstellar parameters in Table 2 (solid line), and the best-fit profile before instrumental smoothing (dashed line).
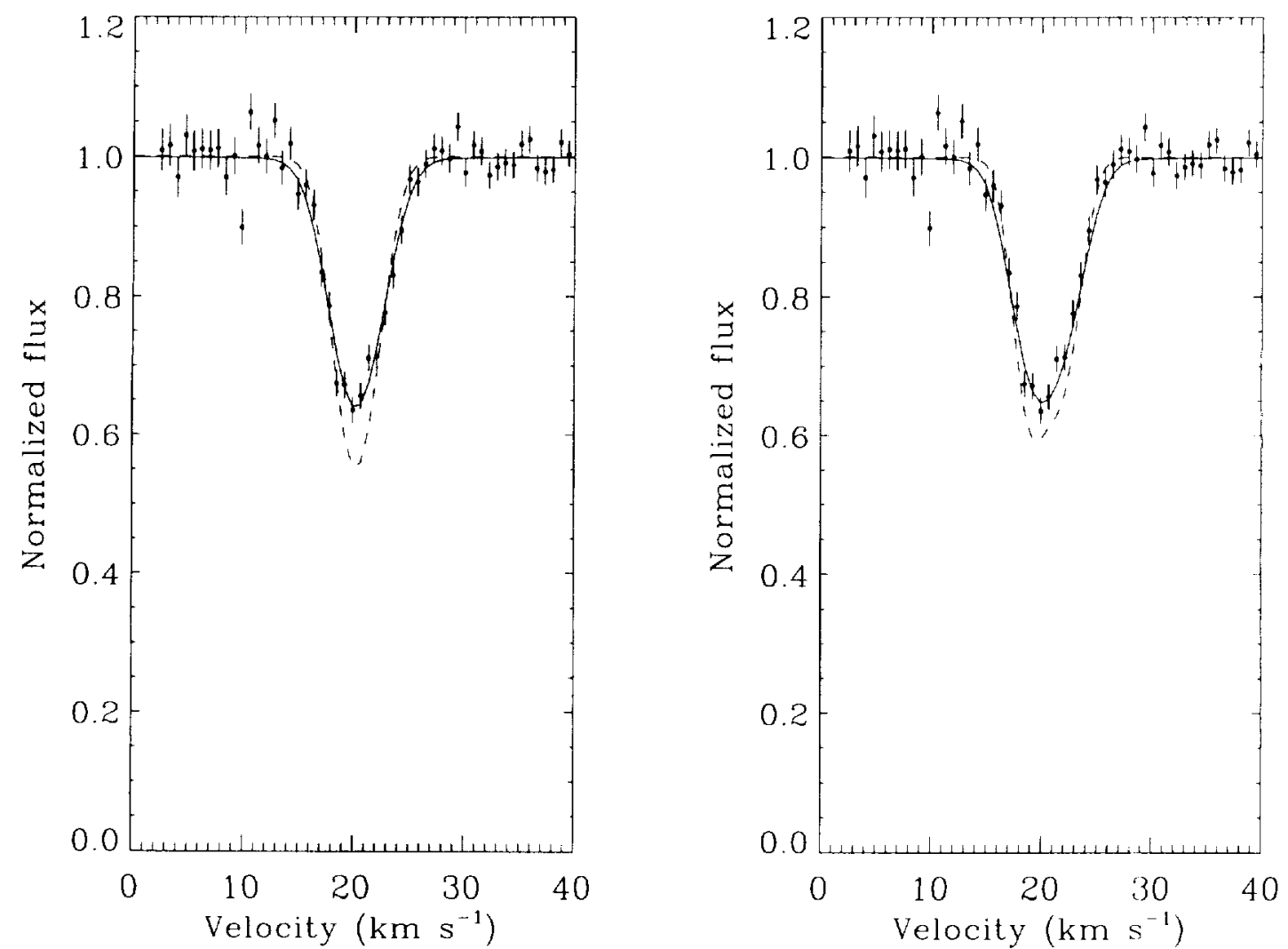

FIG. 8.-Echelle-B spectrum of Procyon's normalized interstellar Fe II $\lambda 2599$ line (with $\pm 1 \sigma$ error bars). Left, best fit to the data using the one velocity component interstellar parameters in Table 2 (solid line), and the best fit before instrumental smoothing (dashed line). Right, same as left panel, but for the two velocity component parameters in Table 3. 

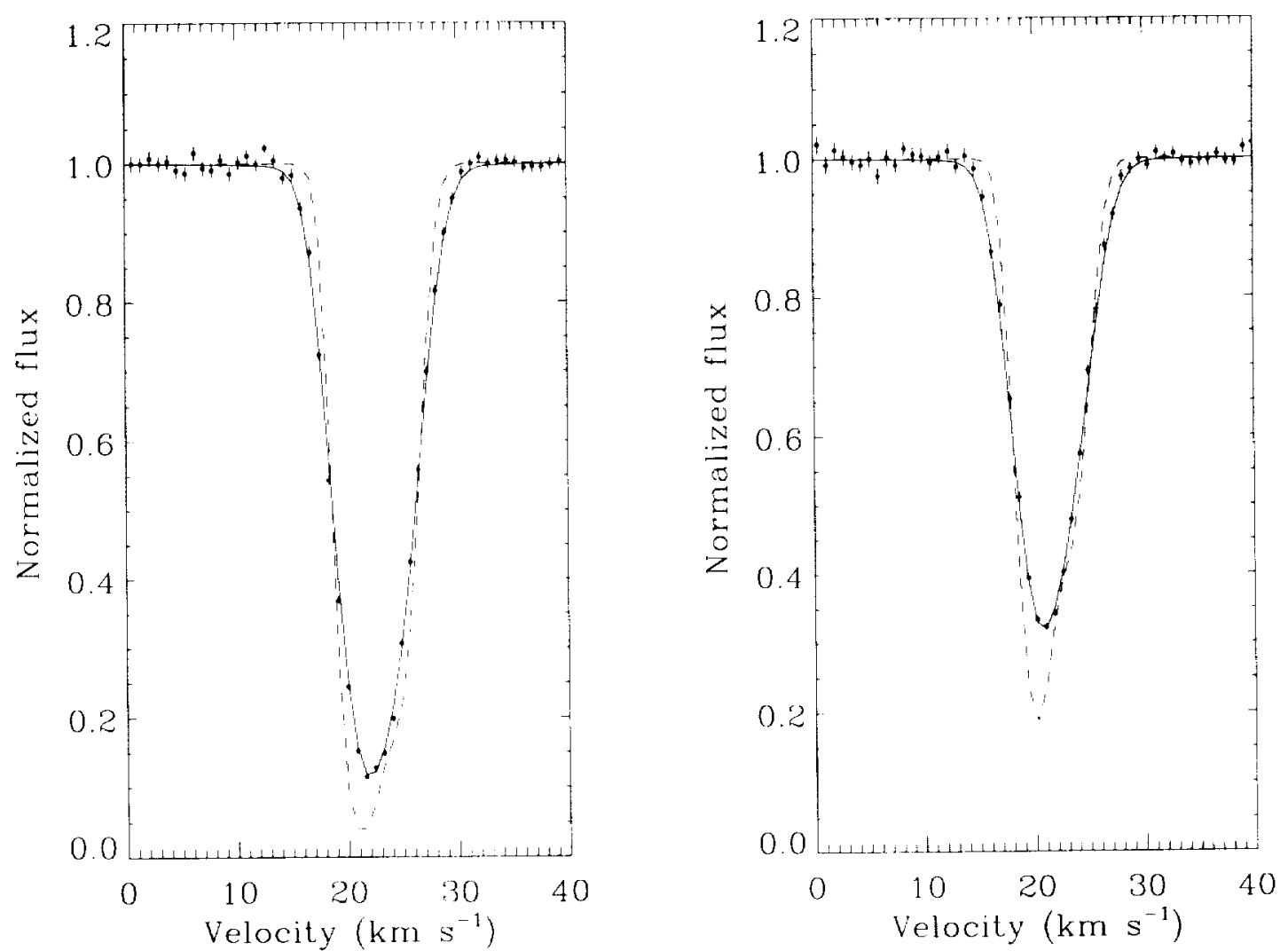

Fig. 9.-Echelle-B spectra of Procyon's normalized interstellar $\mathrm{Mg}$ in $k(2796 \AA)$ (left) and $h(2803 \AA)$ (right) lines (with $\pm 1 \sigma$ error bars), the best fit to the data using the two velocity component interstellar parameters in Table 3 (solid line), and the best fit before instrumental smoothing (dashed line).

component models (col. [4] in Table 2) and two-component models (Table 4) are nearly identical, except that the $b$ values for the two-component models are smaller, especially for the $\mathrm{Mg}$ II and $\mathrm{Fe}$ II lines, and the derived value of $\xi$ is smaller. These results are expected because the instrumental resolution and the widths of the $\mathrm{H}_{\mathrm{I}}$ and $\mathrm{D}_{\mathrm{I}}$ lines are much larger than the velocity separation of the two interstellar components, but for $\mathrm{Mg}$ II and $\mathrm{Fe}$ II the instrumental resolution and line widths are similar to the interstellar velocity separation.

TABLE 4

Two-Component Interstellar Parameters for the Procyon LINE OF SIGHT

\begin{tabular}{|c|c|c|}
\hline Parameter & Component 1 & Component 2 \\
\hline$M g \| k v_{c}\left(k^{\prime} s^{-1}\right)$. & $21.24 \pm 0.10$ & $24.04 \pm 0.10$ \\
\hline $\mathrm{Mg} \| k b\left(\mathrm{~km} \mathrm{~s}^{-1}\right) \ldots \ldots$. & $2.3 \pm 0.10$ & $2.3 \pm 0.10$ \\
\hline $\mathrm{Mg}$ il $k N_{\mathrm{L}}\left(10^{12} \mathrm{~cm}^{-2}\right) \ldots \ldots$ & $2.29 \pm 0.10$ & $1.4 \pm 0.08$ \\
\hline $\mathrm{Mg}$ II $h v_{c}\left(\mathrm{~km} \mathrm{~s}^{-1}\right) \ldots \ldots \ldots \ldots$ & $20.35 \pm 0.10$ & $22.7 \pm 0.10$ \\
\hline $\mathrm{Mg}$ II $h b\left(\mathrm{~km} \mathrm{~s}^{-1}\right) \ldots \ldots \ldots \ldots$ & $2.30 \pm 0.10$ & $2.30 \pm 0.10$ \\
\hline $\mathrm{Mg}$ ॥ा $h N_{L}\left(10^{12} \mathrm{~cm}^{-2}\right) \ldots \ldots$ & $2.29 \pm 0.10$ & $1.2 \pm 0.08$ \\
\hline $\mathrm{Fe} \| v_{c}\left(\mathrm{~km} \mathrm{~s}^{-1}\right) \ldots \ldots \ldots$ & $19.0 \pm 0.10$ & $22.0 \pm 0.10$ \\
\hline Fe II $b\left(\mathrm{~km} \mathrm{~s}^{-1}\right) \ldots \ldots$ & $2.1 \pm 0.10$ & $2.1 \pm 0.10$ \\
\hline Fe II $N_{L}\left(10^{12} \mathrm{~cm}^{-2}\right)$ & $1.12 \pm 0.06$ & $0.88 \pm 0.05$ \\
\hline $\mathrm{H} I v,(\mathrm{~km} \mathrm{~s}-1) \ldots$. & $20.5 \pm 0.10$ & $24.0 \pm 0.10$ \\
\hline H I $b\left(\mathrm{~km} \mathrm{~s}^{-1}\right) \ldots \ldots$ & $10.78 \pm 0.10$ & $10.78 \pm 0.10$ \\
\hline H I $N_{L}\left(10^{18} \mathrm{~cm}^{-2}\right)$ & $0.75 \pm 0.02$ & $0.40 \pm 0.01$ \\
\hline D I $v_{c}\left(\mathrm{~km} \mathrm{~s}^{-\mathrm{I}}\right) \ldots$ & $20.5 \pm 0.10$ & $24.0 \pm 0.10$ \\
\hline $\mathrm{D}$ I $b\left(\mathrm{~km} \mathrm{~s}^{-1}\right) \ldots \ldots$ & $7.59 \pm 0.10$ & $7.59 \pm 0.10$ \\
\hline D) $N_{L}\left(10^{13} \mathrm{~cm}^{-2}\right)$ & $1.19 \pm 0.10$ & $0.64 \pm 0.04$ \\
\hline$T(\mathrm{~K}) \ldots \ldots \ldots \ldots$ & $6900 \pm 80$ & $6900 \pm 80$ \\
\hline$\xi\left(\mathrm{km} \mathrm{s}^{-1}\right)$ & $1.21 \pm 0.27$ & $1.21 \pm 0.27$ \\
\hline
\end{tabular}

We present in column (4) of Table 2 the $\mathrm{H}$ I and D I parameters for the best one-component model. With the assumed broadened solar Ly $\alpha$ line profile, our fit to the shapes of the $\mathrm{H}$ I and $\mathrm{D}_{1}$ line profiles leads to a hydrogen broadening parameter $b_{\mathrm{HI}}=10.83 \pm 0.15 \mathrm{~km} \mathrm{~s}^{-1}$, which is the same as the average value $b_{\mathrm{HI}}=10.87 \pm 0.28 \mathrm{~km} \mathrm{~s}^{-1}$ that we now find for Capella. The thermal $(T=6769 \pm 35 \mathrm{~K})$ contribution to this broadening is similar to the rederived values for the Capella line of sight, but the turbulent contribution $\left(\xi=2.15 \pm 0.12 \mathrm{~km} \mathrm{~s}{ }^{\prime 1}\right)$ is larger.

We have explored a range of parameters $N_{\mathrm{H}}$ and $\mathrm{D} / \mathrm{H}$ to minimize $\chi^{2}$, which characterizes the difference between the observed and simulated Ly $\alpha$ profiles. Figure 11 shows model fits to the observed $D_{\text {I }}$ line assuming the broadened solar emission-line profile and $(\mathrm{D} / \mathrm{H})_{\mathrm{LISM}}=1.2,1.4$, and $1.6 \times 10^{-5}$. None of the fits are particularly good, although they suggest that $(\mathrm{D} / \mathrm{H})_{\mathrm{LISM}}$ lies in the range $1.40 \pm 0.20 \times 10^{-5}$.

Since our assumption that the intrinsic Procyon Ly $\alpha$ line is a broadened solar profile did not lead to an acceptable fit to the observed D I line for any value of $\mathrm{D} / \mathrm{H}$, we instead assumed that the interstellar $\mathrm{D} / \mathrm{H}$ ratio is the same as for the Capella line of sight $\left[(\mathrm{D} / \mathrm{H})_{\mathrm{LISM}}=1.60 \times 10^{-5}\right]$ and modified the shape of the broadened solar line profile to minimize $\chi^{2}$ between the observed line profile and the intrinsic line profile folded through the interstellar medium and the instrument. Figure 12 illustrates our derived intrinsic Ly $\alpha$ profile and compares the observed and simulated line profiles. In Figure 13 we compare the observed line profile to models for $(\mathrm{D} / \mathrm{H})_{\mathrm{LISM}}=1.4,1.6$, and $1.8 \times 10^{-5}$. Now the match to the observed profile is much improved. 


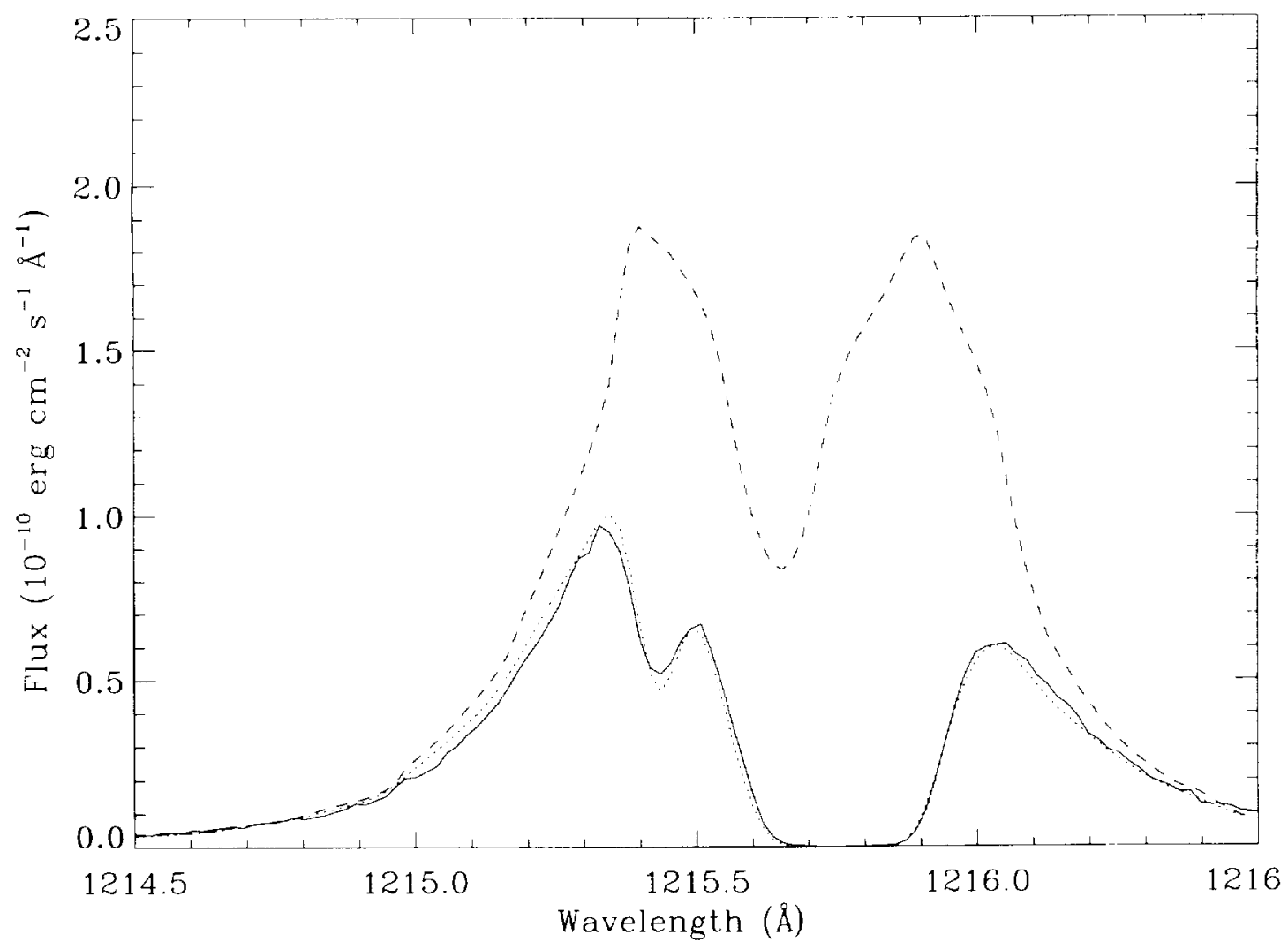

FIG. 10.-Solid line: Observed Ly $\alpha$ spectrum of Procyon. Long dashes: Solar profile broadened about line center by a factor of 1.4. Short dashes: Broadened solar profile folded through the interstellar medium with the parameters in the fourth column of Table 2 including $(\mathrm{D} / \mathrm{H})_{\mathrm{LISM}}=1.40 \times 10^{-5}$.

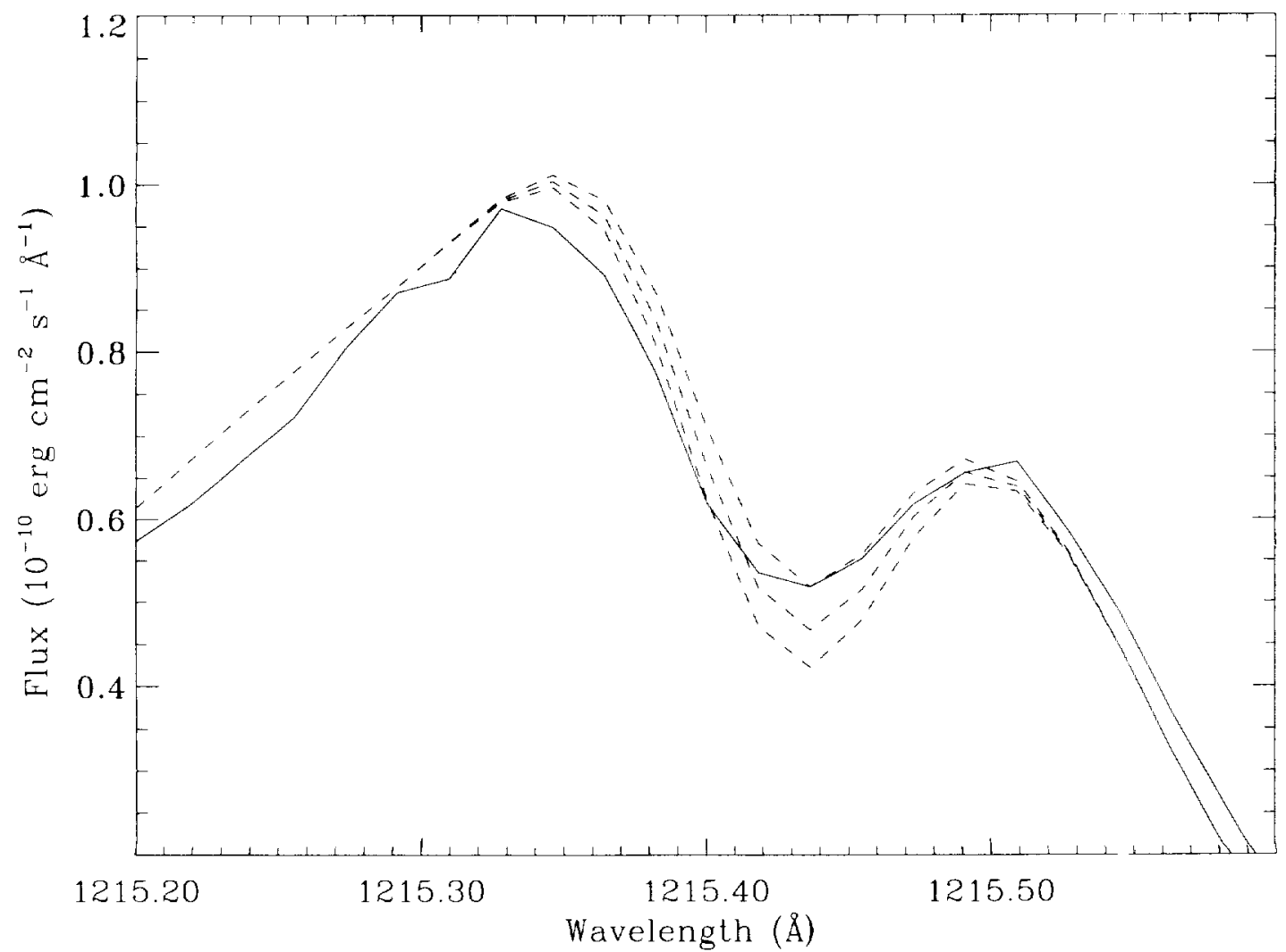

FIG. 11- Expanded view of the deuterium line in Procyon's spectrum. Solid line: Observed spectrum. Dashed lines: Broadened solar profile folded through the interstellar medium with the parameters in the fourth column of Table 2 and (top to bottom) $(\mathrm{D} / \mathrm{H})_{\mathrm{LISM}}=1.2,1.4$, and $1.6 \times 10^{-5}$. 


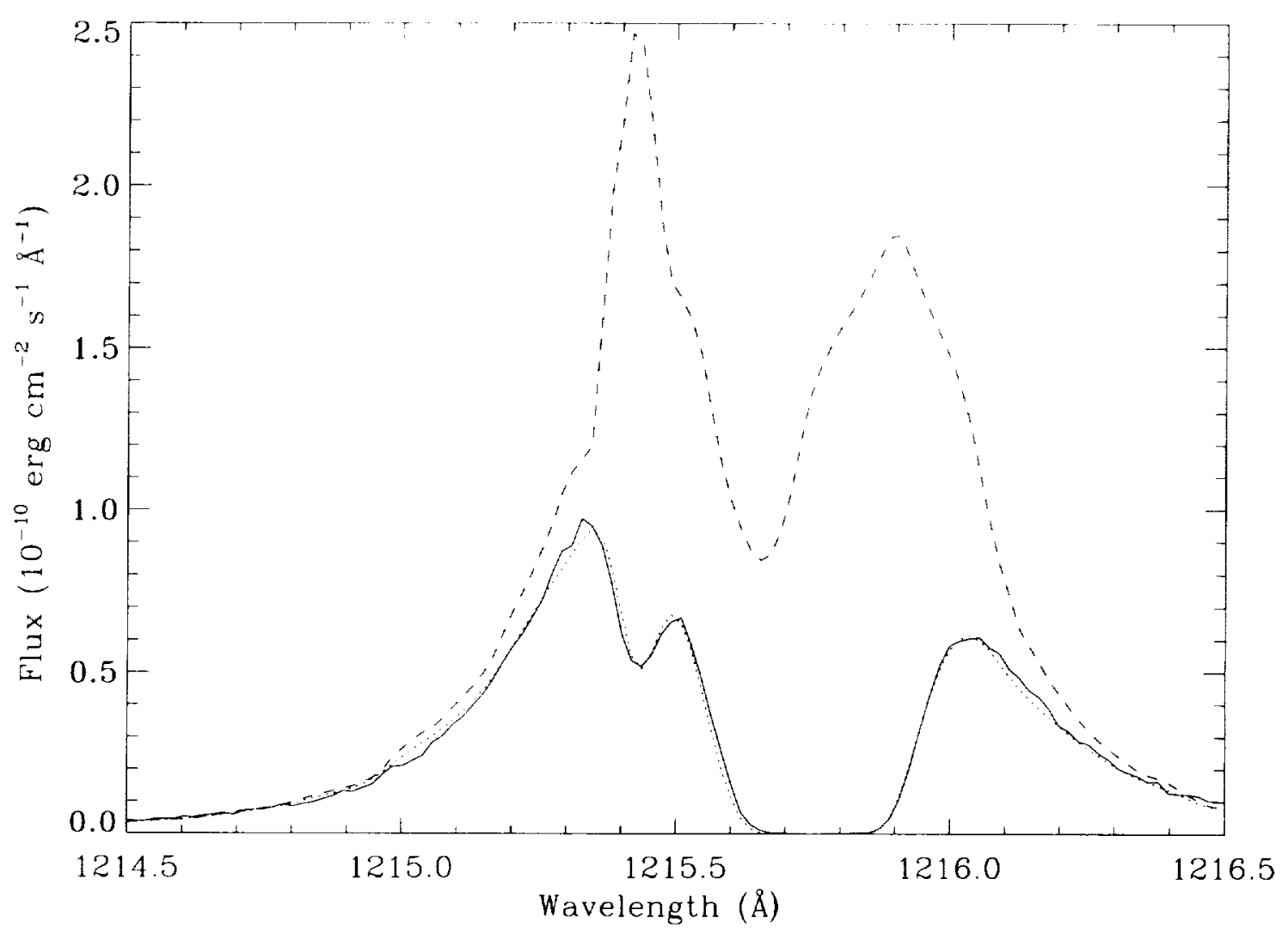

FIG. 12.-Solid line: Observed Ly spectrum of Procyon. Long dashes: Solar profile broadened about line center by a factor of 1.4 and modified by enhancing the blue peak. Short dashes: Modified solar profile folded through the two velocity component interstellar medium with the parameters in Table 3 and $(D / H)_{L I S M}=$ $1.60 \times 10^{-5}$

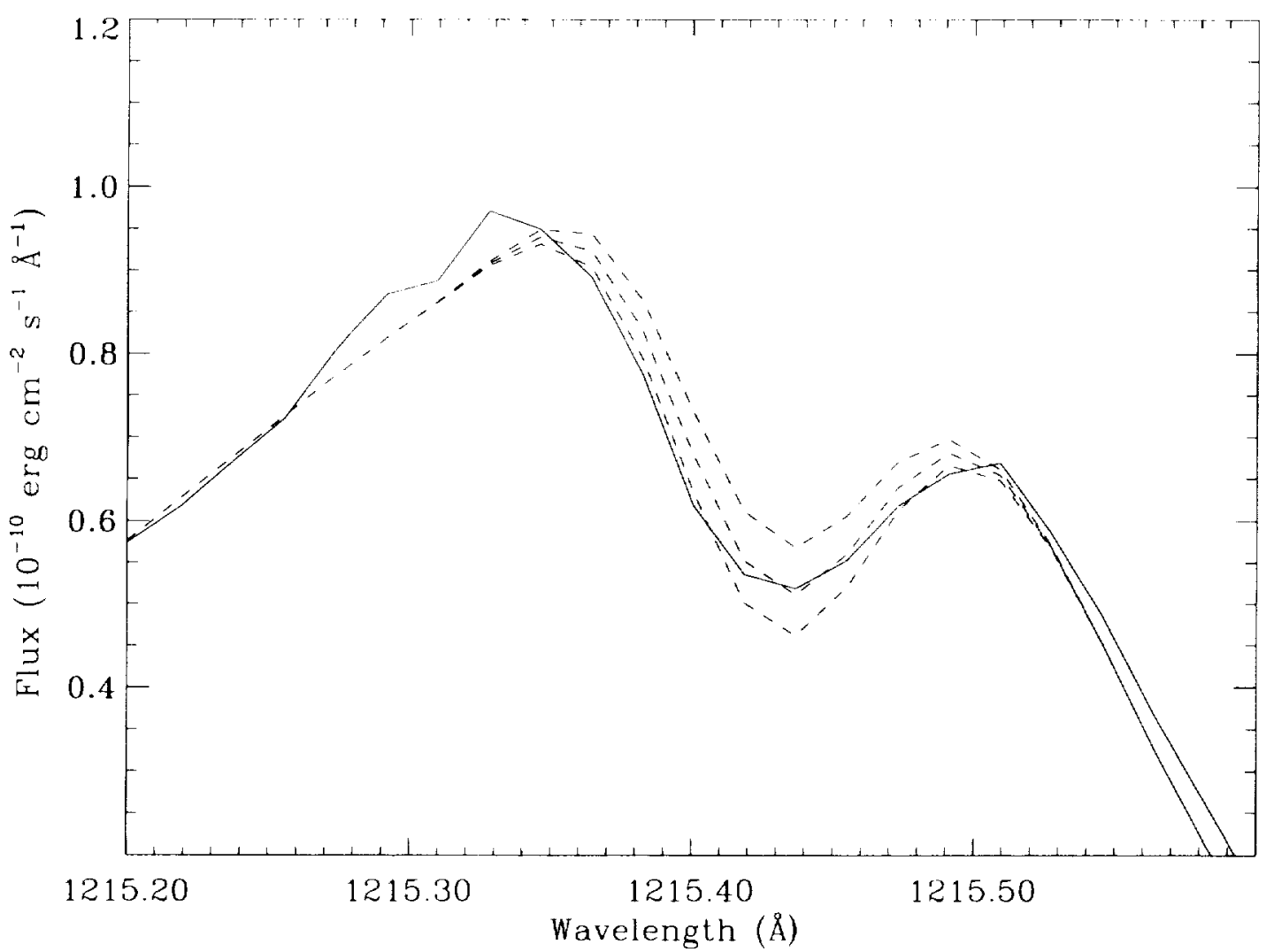

FIG. 13.-Expanded view of the D i line in Procyon's spectrum. Solid line: Observed spectrum. Dashed line: Modified solar profile folded through the two velocity component interstellar medium with the parameters in Table 3 and (from top to bottom) $(\mathrm{D} / \mathrm{H})_{\mathrm{LISM}}=1.4,1.6$, and $1.8 \times 10^{-5}$. 
We consider now whether the asymmetric Lyx profile with a brighter short-wavelength peak is confirmed by any other evidence. Neff et al. (1990) found that the Mg II and Ly $\alpha$ profiles for the G8 III star $\delta$ Lep have the same shape when compared on a common $\Delta \lambda$, not velocity, scale. Ayres et al. (1993) found a similar scaling for the $\mathrm{Mg}$ II and Ly $\alpha$ profiles of the two Capella stars and offered a qualitative explanation for the curious effect based on the work of Gayley (1992). We compare in Figure 14 the modified broadened solar Ly $\alpha$ line with the observed $\mathrm{Mg}$ Il $k$ line plotted on the same $\Delta \lambda$ scale. The remarkable agreement between the two profiles, especially in the region of the $D_{1}$ line, provides evidence that our assumed intrinsic Ly $\alpha$ line profile may be correct. We conclude, therefore, that the Procyon data are consistent with the Capella line-of-sight value $(\mathrm{D} / \mathrm{H})_{\text {LISM }}=1.60 \times 10^{-5}$. Nevertheless, the uncertainty in the intrinsic Ly $\alpha$ profile of Procyon prevents us from firmly concluding that $(\mathrm{D} / \mathrm{H})_{\text {LISM }}$ is constant in the LISM.

The interstellar gas parameters that we derived by using the modified solar profile are listed in the right-hand column of Table 2. They lie within the random errors of the values derived using the broadened solar profile (col. [4] in Table 2) and, except for the values of $b$ and $\xi$, they are similar to the values for the two-component model in Table 4. The $\pm 2 \sigma$ random error uncertainties in $N_{\mathrm{H}}$ and $b_{\mathrm{H}}$ listed in column (5) of Table 2 are derived from contour plots (e.g., Fig. 15) corresponding to different values of $\chi^{2}$ between the observed profile and those simulated with different interstellar parameters but the same assumed stellar emission-line profile. It is difficult to estimate the systematic errors in the interstellar parameters that arise from the uncertain intrinsic $L y x$ profile and the uncertain PSF, but the difference between the values of $T$ derived using the two different Ly $\alpha$ profiles suggests a systematic error of $\pm 300 \mathrm{~K}$. We therefore adopt for the Procyon line of sight the parameters $T=6900 \pm 80( \pm 300$ systematic error) $\mathrm{K}$ and $\xi=1.21 \pm 0.27 \mathrm{~km} \mathrm{~s}^{-1}$.

\section{DISCUSSION}

\subsection{Does D/H Vary in the Local Interstellar Medium?}

Our analysis of the high-quality GHRS spectra of the Capella and Procyon lines of sight are consistent with a single value of $(\mathrm{D} / \mathrm{H})_{\text {LISM }}=1.60 \pm 0.09(+0.05,-0.10$ systematic error) $\times 10^{-5}$ for the local interstellar medium, in agreement with the mean value $\left(1.5 \pm 0.2 \times 10^{-5}\right)$ found by McCullough (1992). There are as yet no other published values of $D / H$ based on GHRS spectra for other lines of sight, although several nearby stars have been observed. We will be extending our study of $\mathrm{D} / \mathrm{H}$ to other lines of sight in upcoming GHRS observations. Eventually, the Far-Ultraviolet Spectroscopic Explorer ( $F U S E$ ) will extend the program to the higher Lyman lines, and thus to larger $\mathrm{H}$ I columns and more distant targets. If real variations in $\mathrm{D} / \mathrm{H}$ are found, then we will have a powerful tool for studying the physical processes that drive the chemical evolution of the Galaxy over short distances and timescales. Bruston et al. (1981) have discussed mechanisms that could explain variations in $\mathrm{D} / \mathrm{H}$ on short distance scales.

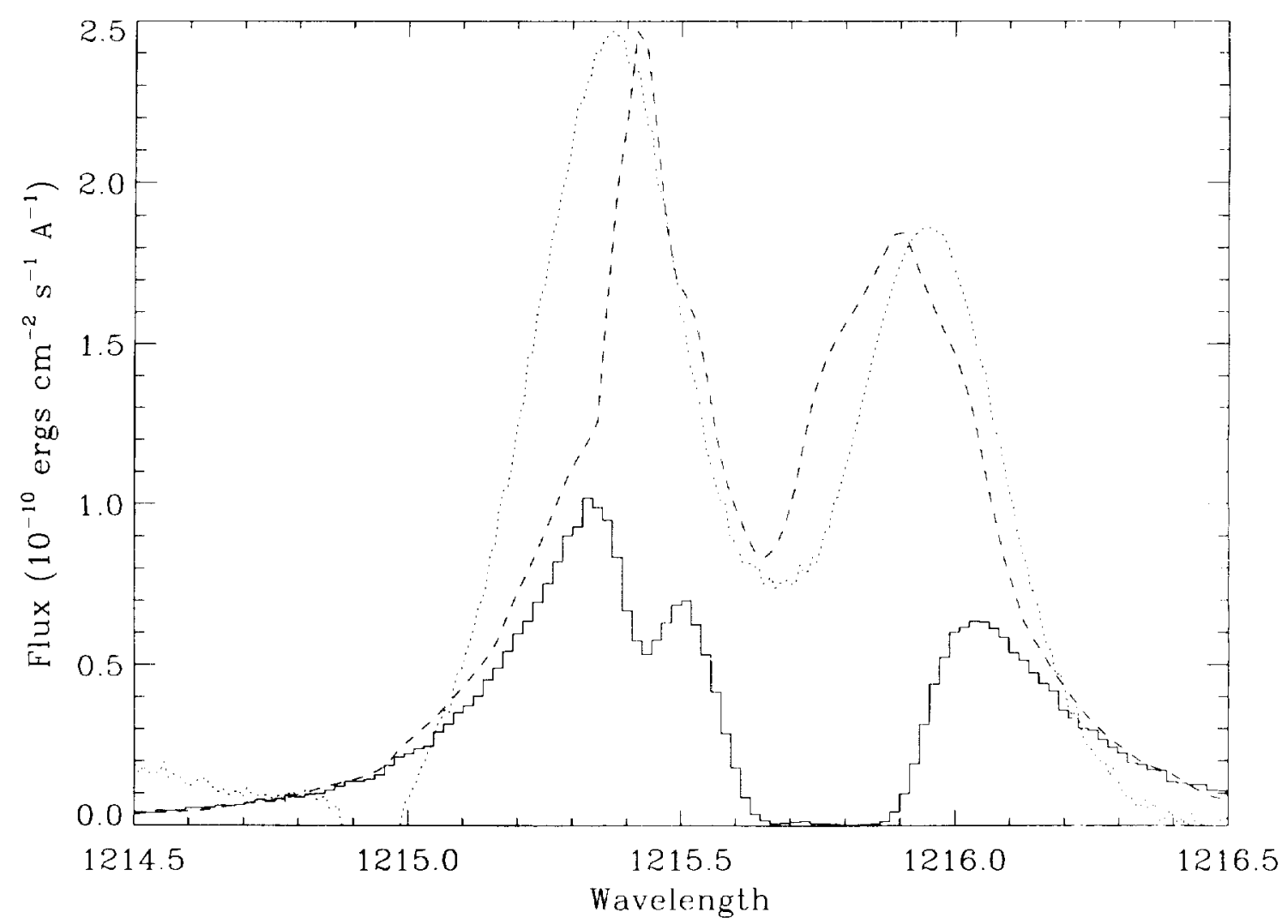

Fig. 14.-Histogram: Observed Lyx profile of Procyon. Long dashes: Modified solar profile. Short dashes: Observed Procyon Mg it $k$ line plotted on the Ly $\alpha$ wavelength scale and scaled to the same peak flux as the modified solar profile. 


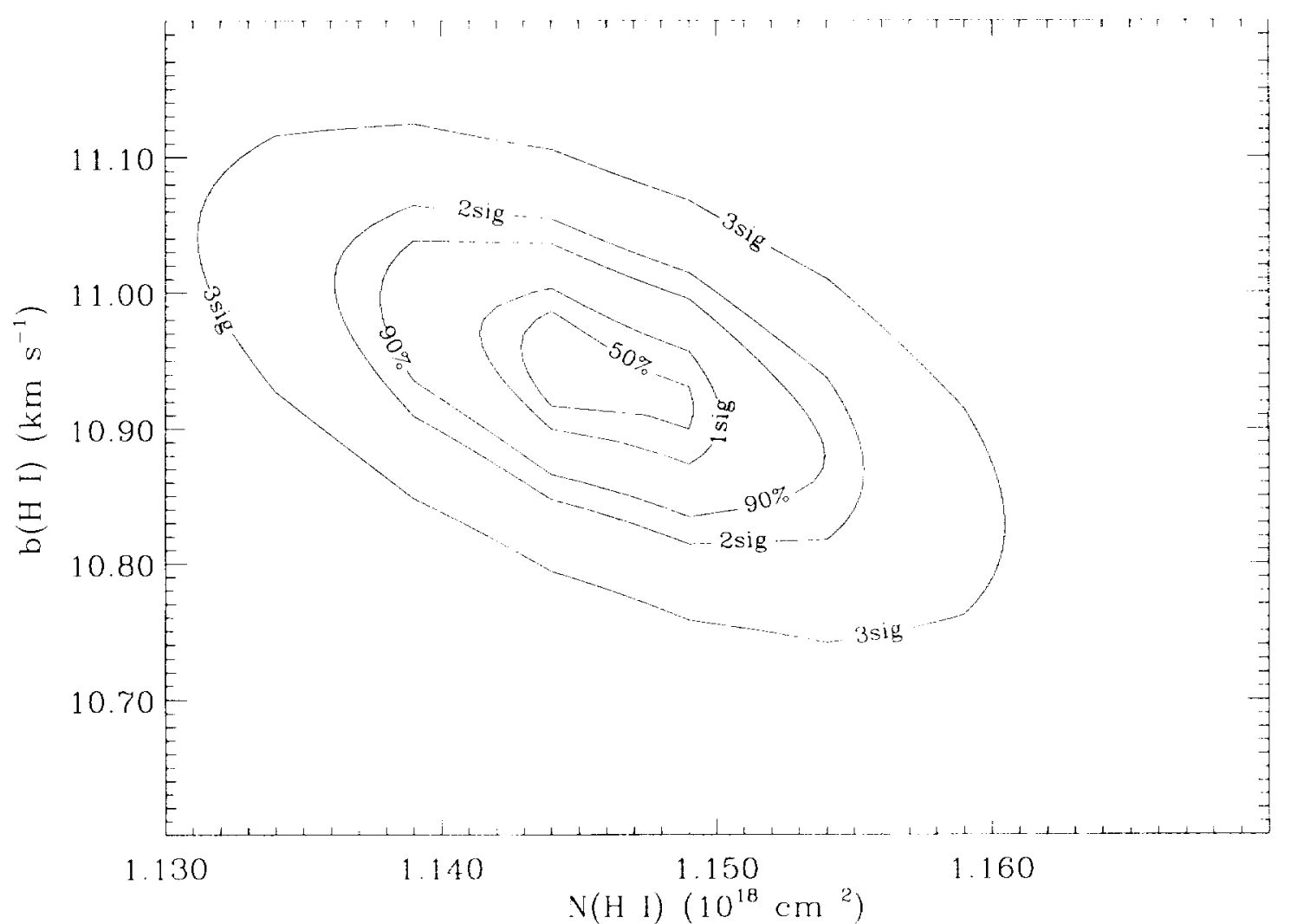

FiG. 15.-Contour plot of $\mathrm{H}$ I column density $N_{\mathrm{H} I}$ vs. hydrogen-broadening parameter $b_{\mathrm{H}}$ to fit the Procyon data by using the modified solar profile.

\subsection{Properties of the Local Interstellar Gas}

Analyses of optical and ultraviolet absorption lines toward nearby stars (including late-type stars, hot white dwarfs, and a few B-type stars), studies of interstellar gas flowing into the heliosphere, and recent studies of EUV absorption toward nearby stars support the currently accepted model for the LISM within the region of space where $N_{\mathrm{H} 1} \leq 10^{19} \mathrm{~cm}^{-2}$. In this model the Sun is located within, but near the edge of, a warm cloud (also called the local cloud or the local "fluff") in which the gas is mostly neutral with $n_{\mathrm{HI}} \approx 0.1 \mathrm{~cm}^{-3}$ and $T \approx 10^{4} \mathrm{~K}$ (see, e.g., Frisch \& York 1983; Bruhweiler \& VidalMadjar 1987; Frisch 1994a, b; Vallerga et al. 1994). The local cloud extends for roughly $60 \mathrm{pc}$ (to $N_{\mathrm{H}} \sim 2 \times 10^{19} \mathrm{~cm}^{-2}$ ) toward the Sco-Cen Association but only $2-3 \mathrm{pc}$ in some other directions. The minimum value of $N_{\mathrm{H} 1}$ lies in the range 0.5 $1.0 \times 10^{18} \mathrm{~cm}^{-2}$ (Vennes et al. 1994). The local cloud is embedded in hot ionized gas $\left(T \sim 10^{6} \mathrm{~K}\right)$ with much lower density $\left(n_{\mathrm{H} 1} \sim 10^{-2.5} \mathrm{~cm}^{-3}\right)$ and separated from it by a thin conductive interface. Other warm clouds are seen in some directions. Thus, lines of sight toward some stars located outside the local cloud show no significant additional $\mathrm{H}$ I column beyond that from the local cloud itself.

In their analysis of high-resolution $\mathrm{Ca}$ II $\mathrm{K}$ line absorption toward six nearby stars, Lallement \& Bertin (1992) derived the flow vector for the local interstellar gas. They found a primary bulk flow velocity of $25.7 \mathrm{~km} \mathrm{~s}^{-1}$ from the direction of Galactic coordinates $l=186^{\circ} .1$ and $b=-16^{\circ} .4$. Two additional absorption components are present along the $5 \mathrm{pc}$ line of sight toward $\alpha$ Agl (Ferlet, Lallement, \& Vidal-Madjar 1986), and one additional component is seen along the $2.7 \mathrm{pc}$ line of sight toward Sirius (Lallement et al. 1994). The Capella spectra show only one velocity component at $22.0 \pm 0.9$ (heliocentric) $\mathrm{km} \mathrm{s}{ }^{1}$, which agrees precisely with the $22.0 \mathrm{~km} \mathrm{~s}^{-1}$ projection of the local flow velocity vector toward Capella. Both $\alpha$ Aql and Sirius also show an absorption component at the projected velocity of the local cloud. For Procyon we find two velocity components: component 1 at $20.8 \pm 1.5 \mathrm{~km} \mathrm{~s}^{-1}$ and component 2 at $23.4 \pm 1.5 \mathrm{~km} \mathrm{~s}^{-1}$, based on the means of the measured velocities of the $\mathrm{Mg} \| h$ and $k$ lines. The $1 \sigma$ error of $\pm 1.5 \mathrm{~km} \mathrm{~s}^{-1}$ is the expected absolute velocity error for GHRS echelle spectra obtained through the SSA with platinum lamp calibration spectra (see Paper I). The velocity errors in Tables 2 and 4 are random measurement errors that do not include systematic effects. We do not use the Fe II data which are much noisier than the $\mathrm{Mg}$ II data. Component 1 is consistent with the $19.8 \mathrm{~km} \mathrm{~s}^{-1}$ projection of the local cloud flow vector found by Lallement \& Bertin (1992). Component 2 is not from the same cloud as the second component found by Lallement et al. (1994) toward Sirius, which is in the same portion of the sky as Procyon, since component 2 is $+2.6 \mathrm{~km} \mathrm{~s}^{-1}$ relative to the local cloud, whereas the second component toward Sirius is $-6.2 \mathrm{~km} \mathrm{~s}^{-1}$ relative to the local cloud.

The kinetic temperature of the warm gas in the local cloud has been estimated by several techniques. Recent in situ measurements of interstellar gas flowing through the heliosphere yield temperatures of $7000 \pm 1000 \mathrm{~K}$ for helium and $8000 \pm 1000 \mathrm{~K}$ for $\mathrm{H}_{\mathrm{I}}$ atoms (see discussion in Lallement \& Bertin 1992 and Paper I). Measurements of absorption-line widths can lead to overestimates of the gas kinetic temperature if unresolved velocity components or turbulence are present. This may explain why Gry, York, \& Vidal-Madjar (1985) reported $T=11,000-12,500 \mathrm{~K}$ for the gas along the line of 
sight toward $\beta \mathrm{CMa}$, which is about $200 \mathrm{pc}$ distant, from their analysis of Copernicus Lyman line absorption spectra.

We believe that our GHRS spectra provide the most accurate temperatures to date for the warm neutral gas of the LISM, because with a spectral resolution of 3.54 and $3.27 \mathrm{~km}$ $\mathrm{s}^{-1}$ for the high-opacity $\mathrm{Mg}$ II and Fe II resonance lines, respectively, we can find only one velocity component for the Capella line of sight and two components toward Procyon. These spectral lines of high-mass ions accurately measure the nonthermal (turbulent) broadening. At the same time, the width of the D I Ly $\alpha$ line and the shape of the $H_{\text {I }}$ absorption close to the saturated core tightly constrain the thermal broadening, especially for the echelle spectrum of Capella. The agreement between the temperature and turbulent velocities for the Capella line of sight, as inferred separately from the echelle and G160M spectra of the Ly $\alpha$ lines (see Table 2), indicates that the lower spectral resolution of the $\mathrm{G} 160 \mathrm{M}$ data does not greatly degrade the accuracy of these results. We find that the gas along the Capella and Procyon lines of sight has the same temperature (about $7000 \mathrm{~K}$ ), but the measured turbulent velocity for the Procyon line of sight is smaller, most likely due to the identification of two bulk velocity components along the line of sight. We propose, therefore, that the Procyon value of $\xi=1.21 \pm 0.27 \mathrm{~km} \mathrm{~s}^{-1}$ is the best choice for the turbulence in the local cloud. This value is consistent with $\xi=1.4$ $(+0.6,-1.4) \mathrm{km} \mathrm{s}^{-1}$ that Lallement et al. (1994) found for the local cloud gas toward Sirius and is consistent with the values $T=7200 \pm 2000 \mathrm{~K}$ and $\xi=2.0 \pm 0.3 \mathrm{~km} \mathrm{~s}^{-1}$ that Gry et al. (1995) recently obtained for component 1 in the line of sight toward $\epsilon$ CMa.

We find that the mean $\mathrm{H}$ I density is a factor of 2.4 larger for the Procyon line of sight $\left(n_{\mathrm{H} I}=0.1065 \pm 0.0028 \mathrm{~cm}^{-3}\right)$ compared to the Capella line of sight $\left(n_{\mathrm{H} 1}=0.0450 \pm 0.0006\right.$ $\mathrm{cm}^{-3}$ ). Since the directions toward Procyon and Capella are not very far apart, we can simply explain the different densities by placing Procyon near the edge of the local cloud and Capella well outside the local cloud. If this geometry is valid, then the derived density toward Procyon provides an accurate measurement of the local cloud density, but the cloud extends only $5.3 \mathrm{pc}$ toward Capella with the remaining $7.2 \mathrm{pc}$ of the line of sight consisting of hot gas with essentially no neutral $\mathbf{H ~} \mathbf{l}$. The existence of two clouds along the line of sight to Procyon makes our determination of the local cloud density and the extension of the local cloud toward Capella uncertain.

\subsection{The Boundary Layer between the Local Cloud and the Hot Interstellar Medium}

Why do Procyon and Sirius, two very nearby stars separated in the sky by only $25^{\circ}$, show second velocity components that differ from each other in radial velocity by $10.4 \mathrm{~km} \mathrm{~s}^{-1}$, while Capella, which is separated from Procyon by $52^{\circ}$, does not show a second velocity component? If we make the sensible assumption that a second velocity component is a result of absorption by gas in a distinct cloud along the line of sight to the star but outside of the local cloud, then we can place constraints on the cloud size. If we place the Sirius cloud close to the $2.7 \mathrm{pc}$ distance of Sirius, then its dimension toward the Procyon line of sight must be less than $1.2 \mathrm{pc}$, or we would see an absorption feature at the appropriate velocity in Procyon's spectrum. Similarly, the Procyon cloud cannot extend more than $1.2 \mathrm{pc}$ in the direction of Sirius, and both clouds cannot extend to the Capella line of sight which is somewhat more distant. This implies that both clouds have dimensions that are roughly $1 \mathrm{pc}$ or smaller and that they lie at or just beyond the edge of the local cloud.

We believe that the GHRS spectra of the three stars provide us with the first glimpse of the boundary layer between the local cloud and the surrounding hot interstellar gas. We speculate that this boundary layer differs greatly from the usual picture of a smooth conductive interface. Instead, the boundary layer appears to be turbulent, consisting of many small cloudlets that are being sheared from the local cloud by shocks or uneven ram pressure as the local cloud and the hot interstellar gas collide. These cloudlets presumably have conductive interfaces with the hot gas and may be destroyed on short timescales due to this heating and shearing to even smaller scales. Additional observations of other nearby stars are needed to confirm this cloudlet boundary layer hypothesis.

\subsection{A Speculation Concerning the Origin of the Nonthermal Motions}

Until now, we have characterized the nonthermal motions in the local cloud as Gaussian "turbulence" that adds quadratically with the thermal motions to explain the observed line broadening. The magnitude of the turbulence is very subsonic which is a little surprising, since it implies that the thermal and turbulent motions are not equilibrated and there are no shock waves in the local cloud. Shocks can be produced easily by cloud-cloud collisions. We consider, therefore, an alternative explanation for the nonthermal line broadening.

The very high $\mathrm{S} / \mathrm{N}$ of our $\mathbf{M g}$ II line spectra provided the opportunity to discover two velocity components along the Procyon line of sight that differ by $2.6 \mathrm{~km} \mathrm{~s}^{-1}$, close to the instrumental resolution of $3.54 \mathrm{~km} \mathrm{~s}^{-1}$. Similar quality data provided no indication of a second velocity component along the Capella line of sight, although additional components at smaller velocity separations are possible. Our cloud model with a single bulk velocity vector is, of course, an idealization. The local cloud may not be rigid, but instead may have a small velocity gradient (or velocity shear) such that over distances of a few parsecs the line-of-sight velocity changes gradually by a few $\mathrm{km} \mathrm{s}^{-1}$. This velocity gradient may be concentrated near the cloud boundary in certain directions. Support for this speculation can be found in the identification by Lallement et al. (1994) of cloud G, located very close to the local cloud with a very similar flow direction and a flow speed that differs by only $3 \mathrm{~km} \mathrm{~s}^{-1}$ from that of the local cloud. They argue that the Sun has already moved most of the way through the local cloud and will next enter cloud $G$. We suggest that the local cloud and cloud $G$ are not isolated entities, but rather parts of the same cloud that contains small velocity gradients. We speculate that the measured nonthermal velocities, which we have called "turbulent," may instead indicate the magnitude of the velocity gradients along the line of sight.

\subsection{Gas-Phase Abundance of $\mathrm{O}$ in the Capella Line of Sight}

In addition to the interstellar $\mathrm{H} \mathrm{I}, \mathrm{D} \mathrm{I}, \mathrm{Fe}$ II, and $\mathrm{Mg}$ II absorption lines discussed in this paper and in Paper I, Linsky et al. (1995) also found interstellar $O_{\mathrm{I}} \lambda 1302.2$ absorption in Capella's spectrum. Since this feature was observed with the G140M grating through the large science aperture (LSA) (see Fig. $4 a$ in Linsky et al. 1995), the spectral resolution is insufficient for the type of detailed spectral synthesis that we used to determine the column densities and broadening parameters for the $\mathrm{H}$ I, $\mathrm{D} \mathrm{I}_{\mathrm{I}} \mathrm{Fe}$ II, and $\mathrm{Mg}$ II lines. Instead, we infer the interstellar oxygen abundance from the measured equivalent width 
of the $\mathrm{O}$ I $\lambda 1302.2$ absorption line, $W_{\mathrm{O} I}=0.052 \pm 0.002 \AA$. The error in $W_{\mathrm{O}}$ is very small because the data have very high $S / N$ and the stellar $\mathrm{O}_{\mathrm{I}}$ emission line, which forms the "continuum" against which the absorption is measured, varies smoothly on either side of the narrow interstellar feature.

Since the spectral resolution does not permit us to measure the $O$ I Doppler parameter directly, we infer $b_{O I}=3.13 \mathrm{~km} \mathrm{~s}^{-1}$ from the temperature and turbulent velocity for the Capella line of sight $\left(T=7000 \mathrm{~K}\right.$ and $\xi=1.6 \mathrm{~km} \mathrm{~s}^{-1}$ ) (see Table 2). We use $f=0.04887$ (Morton 1991) for the oscillator absorption strength of the $\mathrm{O}_{\mathrm{I}}$ line and the curve-of-growth techniques outlined by Spitzer (1978) to calculate an O I column density of $N_{\mathrm{O}_{1}}=8.2 \times 10^{14} \mathrm{~cm}^{-2} \mathrm{O}$ I is strongly coupled to $\mathrm{H}$ I via charge exchange collisions. Therefore, $\mathrm{O} I$ and $\mathrm{H} I$ will exist in the same regions of space, and $N_{\mathrm{O} I} / N_{\mathrm{H}}$ should be a good measure of the gas phase abundance of oxygen. The oxygen abundance is then $\log \left(N_{\mathrm{O}} / N_{\mathrm{H}}\right)=-3.32$, assuming an $\mathrm{H}_{\mathrm{I}}$ column density of $N_{\mathrm{H} I}=1.735 \pm 0.025 \times 10^{18} \mathrm{~cm}^{-2}$ (see Table 2). The solar abundance is $\log (\mathrm{O} / \mathrm{H})_{\odot}=-3.07$ (Anders \& Grevesse 1989), so our measured $O$ abundance represents a logarithmic depletion of $D(\mathrm{O})=\log \left(N_{\mathrm{O}} / N_{\mathrm{H}}\right)-\log (\mathrm{O} / \mathrm{H})_{\odot}=$ -0.25 . The $O$ depletion in the LISM toward Capella is similar to the measurements for other, more distant lines of sight (Jenkins 1987). We also note that the $O$ abundance toward Capella is the same as that measured for B star photospheres (Gies \& Lambert 1992). The issue of choosing the "cosmic" reference abundances for interstellar studies is an important one (see $\$ 4.2$ of Sofia, Cardelli, \& Savage 1994). Additional observations of $\mathrm{O}_{1}$ in the LISM may provide important new insights regarding this problem.

\subsection{Gas-Phase Abundances of Fe and $\mathrm{Mg}$ in the Procyon Line of Sight}

We now estimate the abundances of $\mathrm{Fe}$ and $\mathrm{Mg}$ in the Procyon line of sight by making the same assumptions as in Paper I. For the column densities of $\mathrm{Fe} \mathrm{ll}, \mathrm{Mg}$ II, and $\mathrm{H}$ I we sum the contributions of the two velocity components: $N_{\mathrm{Fe} \mathrm{II}}=2.0 \times 10^{12} \mathrm{~cm}^{-2}, \quad N_{\mathrm{Mg} \text { II }}=3.6 \times 10^{12} \mathrm{~cm}^{-2}$, and $N_{\mathrm{HI}}=1.15 \pm 0.03 \times 10^{18} \mathrm{~cm}^{-2}$, respectively. Therefore, $\log$ $\left(N_{\mathrm{Fe} \mathrm{II}} / N_{\mathrm{HI}}\right)=-5.76$ and $\log \left(N_{\mathrm{Mg} \mathrm{I}} / N_{\mathrm{HI}}\right)=-5.50$. The Fe II abundance for the Procyon line of sight is the same as for Capella, but the $\mathrm{Mg}$ II abundance is 0.06 dex smaller. Comparing these numbers to the abundances of $\mathrm{Fe}$ and $\mathrm{Mg}, \log$ $(\mathrm{Fe} / \mathrm{H})_{\odot}=-4.49$ and $\log (\mathrm{Mg} / \mathrm{H})_{\odot}=-4.41$ from Anders \& Grevesse (1989), and assuming that the contributions to $\mathrm{Fe}$ II and $\mathrm{Mg}$ II from ionized gas are negligible, we derive the logarithmic depletions, $D(\mathrm{Fe})=\left(N_{\mathrm{Fe} \mathrm{III}} / N_{\mathrm{HI}}\right)-\log (\mathrm{Fe} / \mathrm{H})_{\odot}=$ -1.27 and $D(\mathrm{Mg})=\left(N_{\mathrm{Mg}_{\mathrm{g}} \mathrm{V}} / N_{\mathrm{H} \mathrm{J}}\right)-\log (\mathrm{Mg} / \mathrm{H})_{\odot}=-1.09$ in the warm gas toward Procyon.

These $\mathrm{Mg}$ and $\mathrm{Fe}$ depletion values can be compared to Copernicus estimates of $D(\mathrm{Mg})=-0.3$ and $D(\mathrm{Fe})=-1.4$ in the warm interstellar medium (Jenkins, Savage, \& Spitzer 1986). The value for $\mathrm{Fe}$ is similar to that measured for the Capella and Procyon sight lines, while the value for $\mathbf{M g}$ differs by $0.8 \mathrm{dex}$. Most of the difference may be due to a systematic error in the $f$-values for the far-ultraviolet $\mathbf{M g}$ II lines near 1240 $\AA$ used in the Copernicus study. Sofia et al. (1994) found from a careful analysis of GHRS echelle mode data for interstellar gas toward $\xi$ Per that the far-UV lines of $\mathbf{M g}$ II yield $\mathbf{M g}$ II column densities 0.67 dex larger than those obtained from an analysis of the strong but damped $\mathrm{Mg}$ II lines near $2800 \AA$. Since the $f$-values of the $\mathrm{Mg}$ II $\lambda 2800$ lines are well determined, they recommended a 0.67 dex revision to the $f$-values for the $\mathrm{Mg}$ II
far-UV doublet. Although the $\mathrm{Mg}$ II results may be uncertain, the measurements for Fe II clearly reveal that substantial gasphase depletion occurs in the cloud toward Capella and Procyon. Evidently the $\mathrm{Fe}$ in the cloud is mostly found in interstellar dust.

\section{COSMOLOGICAL SIGNIFICANCE OF THESE RESULTS}

As described in $\S 1$, an important objective of our program is to measure $\mathrm{D} / \mathrm{H}$ in the LISM and to infer the primordial number ratio $(\mathrm{D} / \mathrm{H})_{p}$, which tells us the number density of baryons during the period 100-1000 s after the big bang when the universe had cooled enough for the light nuclei to form. Since the Hubble expansion relates the baryon densities then and now, $(\mathrm{D} / \mathrm{H})_{p}$ also determines the mean baryon density in the universe today and the ratio $\Omega_{B}$ of the local baryon density to the critical density (the required density to eventually halt the expansion). Thus, $(\mathrm{D} / \mathrm{H})_{p}$ is an important parameter for experimental cosmology.

Although our data do not allow us to determine $(\mathrm{D} / \mathrm{H})_{p}$ directly, we can infer its value from our measurement of $(\mathrm{D} / \mathrm{H})_{\text {LISM }}$ and chemical evolution models for the Galaxy. Steigman \& Tosi (1992), for example, have calculated the survival fraction of deuterium as the primordial $D$ is converted to heavier elements in the cores of stars and the deuteriumdepleted gas is dispersed into the interstellar medium from which later generations of stars are formed. Their calculations indicate that $(\mathrm{D} / \mathrm{H})_{p}=1.5-3.0 \times(\mathrm{D} / \mathrm{H})_{\mathrm{LISM}}$, so that $(\mathrm{D} / \mathrm{H})_{p}=$ $2.3-5.1 \times 10^{-5}$. On the other hand, Vangioni-Flam, Olive, \& Prantzos (1994) have argued that an astration factor (the inverse of the survival fraction) of about 5 is more reasonable, in which case $(\mathrm{D} / \mathrm{H})_{p} \approx 8 \times 10^{-5}$. Comparison of the value for $(\mathrm{D} / \mathrm{H})_{p}$, assuming the Steigman-Tosi astration factor of 1.5-3.0 with recent big bang nucleosynthesis calculations (Walker et al. 1991), indicates that $\eta_{10}=3.5-5.8$, where $\eta_{10}$ is $10^{10}$ times the ratio of nucleons to photons by number. The range in $\eta_{10}$ leads to the very important result that $0.05 \leq \Omega_{B} h_{50}^{2} \leq 0.09$, where $h_{50}^{2}$ is the Hubble constant in units of $50 \mathrm{~km} \mathrm{~s}^{-1} \mathrm{Mpc}^{-1}$. The Vangioni-Flam et al. astration factor instead leads to $\eta_{10} \approx 2.8$ and $\Omega_{B} h_{50}^{2} \approx 0.042$. Thus, no matter what value one assumes for the Hubble constant, $\Omega_{B} \ll 1$, and a universe with a cosmological constant of zero consisting only of baryons must be very open. Tremaine (1992) and others, however, argued that $\approx 90 \%$ of the universe consists of dark nonbaryonic matter on the basis of the dynamical properties of galaxies and clusters. Thus, whether $\Omega \geq 1.0$ is true remains an open question.

Another important result in experimental cosmology is that our lower limit on $(\mathrm{D} / \mathrm{H})_{\text {LISM }} \geq 1.41 \times 10^{-5}$ provides a hard lower limit to $(\mathrm{D} / \mathrm{H})_{p}$ and thus a hard upper limit on $\eta_{10} \leq 7.8$ and $\Omega_{B} h_{30}^{2} \leq 0.125$. These limits are independent of Galactic chemical evolution models and only assume that $D$ is destroyed with time.

\section{CONCLUSIONS}

In this second in our series of studies of the $\mathrm{D} / \mathrm{H}$ ratio and other properties of warm interstellar gas along the lines of sight toward nearby stars, we analyze GHRS spectra toward Procyon (3.5 pc) and Capella (12.5 pc). Observations of Capella near opposite orbital quadratures (orbital phases 0.26 and 0.80 ) permit us to derive both the intrinsic stellar Ly $\alpha$ line profiles and the interstellar absorption lines more precisely than before. We conclude that $(\mathrm{D} / \mathrm{H})_{\text {LISM }}=1.60 \pm 0.09(+0.05$, -0.10 systematic error) $\times 10^{-5}$. Our analysis of the Lya, 
$\mathrm{Mg}$ II, and $\mathrm{Fe}$ II lines indicates that the temperature of the interstellar gas is $T=7000 \pm 500$ ( \pm 400 systematic error) $\mathrm{K}$ and the microturbulence is $\xi=1.6 \pm 0.4( \pm 0.2$ systematic error) $\mathrm{km} \mathrm{s}^{-1}$.

Procyon's Mg it line profiles demonstrate that there are two velocity components in that line of sight separated by $2.6 \mathrm{~km}$ $\mathrm{s}^{-1}$, slightly less than the instrumental resolution. Since we could not fit the observed D I line shape by assuming that the intrinsic Ly $\alpha$ line profile is simply a broadened solar profile for any value of $\mathrm{D} / \mathrm{H}$, we assumed that the $\mathrm{D} / \mathrm{H}$ ratio is the same as for the Capella line of sight and modified the broadened solar profile to achieve excellent agreement between the simulated and observed line profiles. The resulting asymmetric intrinsic stellar line profile is similar to the scaled $\mathrm{Mg}$ u profile shape, which supports our choice of this Ly $\propto$ profile. The Procyon data thus provide a consistency check on the Capella value for $(\mathrm{D} / \mathrm{H})_{\mathrm{LISM}}=1.6 \times 10^{-5}$, but the uncertainty in the intrinsic Ly $\alpha$ emission-line profile does not permit us to conclude that the $\mathrm{D} / \mathrm{H}$ ratio is constant in the LISM. Observations of other lines of sight are needed to determine whether a single value of $\mathrm{D} / \mathrm{H}$ characterizes the LISM.

We find that interstellar gas properties of the lines of sight toward Procyon and Capella are very similar. Since the mean $\mathrm{H}$ I density is a factor of 2.4 larger for the Procyon line of sight than for Capella, we propose that the first $5.3 \mathrm{pc}$ along the Capella line of sight lies within the local cloud, while the remaining $7.2 \mathrm{pc}$ lies in the hot gas surrounding the local cloud.
This model leads us to propose that $n_{\mathrm{HI}}=0.1065 \pm 0.0028$ $\mathrm{cm}^{-3}$ be adopted for the neutral hydrogen density within the local cloud and $\xi=1.21 \pm 0.27 \mathrm{~km} \mathrm{~s}^{-1}$ be adopted for the nonthermal motions. We speculate, however, that the measured nonthermal motions which we call "turbulence" are instead indicative of systematic velocity shear within the local cloud: it is not a rigid comoving structure. We find that the depletions of $\mathrm{Fe}$ and $\mathrm{Mg}$ for both lines of sight are at the lower end of the distribution for stars, as is the depletion of $\mathrm{O}$ along the Capella line of sight.

Using the Galactic chemical evolution calculations for D by Steigman \& Tosi (1992) or by Vangioni-Flam et al. (1994), we infer that $0.05 \leq \Omega_{B} h_{50}^{2} \leq 0.09$ or $\Omega_{B} h_{50}^{2} \approx 0.042$, respectively. Thus, no matter what value one assumes for the Hubble constant, we conclude that $\Omega_{B} \ll 1$ and that a universe with a cosmological constant of zero consisting only of baryons must be very open. The assumption of no Galactic chemical evolution leads to a model-independent upper bound to $\Omega_{B} h_{50}^{2} \leq$ 0.125 .

This work is supported by NASA Interagency Transfer S56500-D to the National Institute of Standards and Technology and NASA Grants NAG 5-1858 and NAG 5-1852 to the University of California at San Diego and to the University of Wisconsin-Madison, respectively. We thank the referee for very helpful comments on the manuscript.
Anders, E., \& Grevesse, N. 1989, Geochim. Cosmochim. Acta, 53, 197 Anderson, R. C. 1979, Ph.D. thesis, Johns Hopkins Univ.

Anderson, R. C., Henry, R. C., Moos, H. W., \& Linsky, J. L. 1978, ApJ, 226, 883 Ayres, T. R. 1984, ApJ, 284, 784

A 1988, ApJ, 331, 467

Ayres, T. R., Brown, A., Gayley, K. G., \& Linsky, J. L. 1993, ApJ, 402, 710

Boesgaard, A. M., \& Steigman, G. 1985, ARA\&A, 23, 319

Brandt, J. C., et al. 1994, PASP, 106, 890

Bruhweiler, F. C., \& Vidal-Madjar, A. 1987, in Exploring the Universe with the

IUE Satellite, ed. Y. Kondo (Dordrecht: Reidel), 467

Bruston, P., Audouze, J., Vidal-Madjar, A., \& Laurent, C. 1981, ApJ, 243, 161

Cardelli, J. A., Ebbets, D. C., \& Savage, B. D. 1990, ApJ, 365, 789

Dupree, A. K., Baliunas, S. L., \& Shipman, H. L. 1977, ApJ, 218, 361

Ferlet, R., Lallement, R., \& Vidal-Madjar, A. 1986, A\&A, 163, 204

Frisch, P. C. 1994a, Science, 265, 1423

1994b. Space Sci. Rev, in press

Frisch, P. C., \& York, D. G. 1983, ApJ, 271, L59

Gayley, K. G. 1992, ApJ, 390, 573

Geiss, J., \& Reeves, H. 1981, A\&A, 93, 189

Gies, D. R., \& Lambert, D. L. 1992, ApJ, 387, 673

Gry, C., Lemonon, L., Vidal-Madjar, A., Lemoine, M., \& Ferlet, M. 1995, $A \& A$, in press

Gry, C., York, D. G., \& Vidal-Madjar, A. 1985, ApJ, 296, 593

Heap, S. R., et al. 1995, PASP, submitted

Jenkins, E. B. 1987, in Interstellar Processes, ed. D. H. Hollenbach \& H. A Thronson, Jr. (Dordrecht : Reidel), 533

Jenkins, E. B., Savage, B. D., \& Spitzer, L. 1986, ApJ, 301, 355

Lallement, R., \& Bertin, P. 1992, A\&A, 266, 479

Lallement, R., Bertin, P., Ferlet, R., Vidal-Madjar, A., \& Bertaux, J. L. 1994, A\&A, 286, 898

\section{REFERENCES}

Landsman, W., \& Simon, T. 1993, ApJ, 408, 302

Linsky, J. L., et al. 1993, ApJ, 402, 694 (Paper I)

Linsky, J. L., Wood, B. E., Judge, P., Brown, A., Andrulis, C., \& Ayres, T. R. 1995, ApJ, 442, 381

McClintock, W., Henry, R. C., Linsky, J. L., \& Moos, H. W. 1978, ApJ, 225, 465

McCullough, P. R. 1992, ApJ, 390, 213

Morton, D. C. 1991, ApJS, 77, 119

Murthy, J., Henry, R. C., Moos, H. W., Landsman, W. B., Linsky, J. L., VidalMadjar, A., \& Gry, C. 1987, ApJ, 315, 675

Murthy, J., Henry, R. C., Moos, H. W., Vidal-Madjar, A., Linsky, J. L., \& Gry, C. 1990, ApJ, 356, 223

Neff, J. E., Landsman, W. B., Bookbinder, J. A., \& Linsky, J. L. 1990, in Evolution in Astrophysics, ed. E. J. Rolfe (ESA SP-310), 341

Soderblom, D. 1993, HST Goddard High Resolution Spectrograph Instrument Handbook, Version 4.0, Space Telescope Science Institute

Sofia, U. J., Cardelli, J. A., \& Savage, B. D. 1994, ApJ, 430, 650

Spitzer, L. 1978, Physical Processes in the Interstellar Medium (New York: Wiley-Interscience)

Spitzer, L., Jr., \& Fitzpatrick, E. L. 1993, ApJ, 409, 299

Steigman, G., \& Tosi, M. 1992, ApJ, 401, 150

Tremaine, S. 1992, Phys. Today, 45, 28

Vallerga, J. V., Vedder, P. W., Craig, N., \& Welsh, B. Y. 1994, ApJ, 411, 729

Vangioni-Flam, E., Olive, K. A., \& Prantzos, N. 1994, ApJ, 427, 618

Vennes, S., et al. 1994, ApJ, 421, L35

Vidal-Madjar, A., Laurent, C., Bruston, P., \& Audouze, J. 1978, ApJ, 223, 589

Walker, T. P., Steigman, G., Schramm, D. N., Olive, K. A., \& Kang, H.-S. 1991, ApJ, 376, 51

Wilson, T. L., \& Rood, R. T. 1994, ARA\&A, 32, 191

Wood, B. E., \& Ayres, T. R. 1995, ApJ, 443, 329 
